\title{
Congenic expression of poly-GA but not poly-PR in mice triggers selective neuron loss and interferon responses found in C9orf72 ALS
}

\author{
Katherine D. LaClair ${ }^{1}$ Q Qihui Zhou ${ }^{1,2} \cdot$ Meike Michaelsen $^{1} \cdot$ Benedikt Wefers $^{1} \cdot$ Monika S. Brill $^{2,3} \cdot$ Aleksandar Janjic $^{4}$. \\ Birgit Rathkolb ${ }^{5,6,7}$. Daniel Farny ${ }^{1} \cdot$ Mikolaj Cygan ${ }^{1}$ - Martin Hrabe de Angelis ${ }^{5,7,8}$. Wolfgang Wurst ${ }^{1,2}$. \\ Manuela Neumann ${ }^{9,10} \cdot$ Wolfgang Enard ${ }^{4} \cdot$ Thomas Misgeld $^{1,2,3} \cdot$ Thomas Arzberger $^{1,2,11,12} \cdot$ Dieter Edbauer $^{1,2,13}$ (D)
}

Received: 22 April 2020 / Revised: 4 June 2020 / Accepted: 4 June 2020 / Published online: 19 June 2020

(c) The Author(s) 2020

\begin{abstract}
Expansion of a $\left(\mathrm{G}_{4} \mathrm{C}_{2}\right)_{n}$ repeat in $C 9$ orf 72 causes amyotrophic lateral sclerosis (ALS) and frontotemporal dementia (FTD), but the link of the five repeat-encoded dipeptide repeat (DPR) proteins to neuroinflammation, TDP-43 pathology, and neurodegeneration is unclear. Poly-PR is most toxic in vitro, but poly-GA is far more abundant in patients. To directly compare these in vivo, we created congenic poly-GA and poly-PR mice. $40 \%$ of poly-PR mice were affected with ataxia and seizures, requiring euthanasia by 6 weeks of age. The remaining poly-PR mice were asymptomatic at 14 months of age, likely due to an $80 \%$ reduction of the transgene mRNA in this subgroup. In contrast, all poly-GA mice showed selective neuron loss, inflammation, as well as muscle denervation and wasting requiring euthanasia before 7 weeks of age. In-depth analysis of peripheral organs and blood samples suggests that peripheral organ failure does not drive these phenotypes. Although transgene mRNA levels were similar between poly-GA and affected poly-PR mice, poly-GA aggregated far more abundantly than poly-PR in the CNS and was also found in skeletal muscle. In addition, TDP-43 and other disease-linked RNA-binding proteins co-aggregated in rare nuclear inclusions in the hippocampus and frontal cortex only in poly-GA mice. Transcriptome analysis revealed activation of an interferon-responsive pro-inflammatory microglial signature in end-stage poly-GA but not poly-PR mice. This signature was also found in all ALS patients and enriched in C9orf72 cases. In summary, our rigorous comparison of poly-GA and poly-PR toxicity in vivo indicates that poly-GA, but not poly-PR at the same mRNA expression level, promotes interferon responses in C9orf72 disease and contributes to TDP-43 abnormalities and neuron loss selectively in disease-relevant regions.
\end{abstract}

Keywords $C 9 \operatorname{orf72} \cdot$ ALS $\cdot$ FTD $\cdot$ Neurodegeneration $\cdot$ Mouse model $\cdot$ Microglia $\cdot$ Interferon

\section{Introduction}

Amyotrophic lateral sclerosis (ALS) and frontotemporal dementia (FTD) are fatal neurodegenerative diseases with overlapping clinical symptoms, neuropathological findings and genetic causes. Predominantly cytoplasmic aggregates of the nuclear RNA-binding protein TDP-43 are a key

Katherine D. LaClair and Qihui Zhou contributed equally.

Electronic supplementary material The online version of this article (https://doi.org/10.1007/s00401-020-02176-0) contains supplementary material, which is available to authorized users.

Dieter Edbauer

dieter.edbauer@dzne.de

Extended author information available on the last page of the article feature that correlates with regional neurodegeneration and neuroinflammation [37, 47]. About $10 \%$ of all ALS/FTD patients carry a pathogenic $\left(\mathrm{G}_{4} \mathrm{C}_{2}\right)_{n}$ repeat expansion in the C9orf72 gene. The sense and antisense repeat transcripts are translated in all reading frames into five aggregating dipeptide repeat proteins (DPRs: poly-GA, -GP, -GR, -PA and -PR) in an unconventional process termed repeat-associated non-ATG (RAN) translation [1, 45, 46, 70]. In patients, the DPR proteins co-aggregate in neuronal cytoplasmic inclusions and occasionally in neuronal nuclear inclusions [58]. DPR inclusions are also found in skeletal muscle of $40 \%$ of patients [9]. Poly-GA, translated from the sense strand, forms the most abundant DPR inclusions in C9orf72 carriers, found in all neocortical regions, hippocampus and cerebellum, and to a lesser degree in subcortical regions, brainstem and the spinal cord $[37,38,58]$. In contrast, poly-PR 
is translated from the antisense strand and inclusions are about 100-fold less abundant than for poly-GA in patients [38], but it is clearly the most toxic in cellular models [31, $62,63]$. Furthermore, in cellular models, poly-GA expression has been most directly linked to TDP-43 aggregation $[28,29,48]$. In addition, sense and antisense repeat RNA accumulate in ubiquitous nuclear foci that may interact with a plethora of RNA-binding proteins with high affinity for $\left(\mathrm{G}_{4} \mathrm{C}_{2}\right)_{n}$ RNA, e.g., hnRNP H [8], which may affect splicing and gene expression. Finally, the mutant $C 9$ orf 72 allele is poorly expressed, resulting in widespread reduction of C9orf72 protein expression by $20-40 \%[16,55]$. However, even complete $C 9$ orf 72 knockout does not cause ALS/FTD like phenotypes in mice [23], arguing against loss-of-function as a major contributor to disease. Importantly, none of the $C 9$ orf72-specific phenomena clearly correlate with TDP43 pathology or neuron loss in patients, arguing for complex synergistic effects and/or selective vulnerability [14].

Among BAC transgenic C9orf72 models that produce both repeat RNA and DPRs, some have shown subtle behavioral phenotypes and neuron loss, but the relative contributions of RNA and DPRs cannot be determined [23, 49, 53]. Only one $C 9$ orf72 BAC line shows an aggressive ALS-like phenotype, but only in a subset of female mice [34]. Models that express translation-incompetent repeat RNA or individual DPRs have begun to unravel their individual contributions in C9orf72 disease [6, 7, 20, 23, 34, 43, 49, 53, 57, 66-68]. High-level viral expression of $\left(\mathrm{G}_{4} \mathrm{C}_{2}\right)_{\mathrm{n}}$ RNA, polyGA, poly-GR, or poly-PR cause widespread DPR pathology and variable neurodegeneration by 3-6 months [6, 66-68]. For transgenic lines, homozygous poly-PR-expressing mice so far showed the most severe toxicity due to Purkinje cell loss leading to cerebellar ataxia, but not clearly ALS-like phenotypes [7, 20,57]. Together, this body of work suggests that poly-GA, poly-GR, and poly-PR can all be toxic to the CNS in vivo if the expression levels are high enough. However, the expression strategy and repeat lengths are different for each model, precluding rigorous comparison of the relative contributions of specific DPRs to disease phenotypes and pathomechanisms.

Thus, we generated and characterized novel conditional mouse lines for high-level genomic expression of individual DPR proteins utilizing alternate codon sequences to avoid effects of $\left(\mathrm{G}_{4} \mathrm{C}_{2}\right)_{n}$ RNA toxicity and avoid any potential effects of differential RAN translation efficiency. We focused on the most abundant and the most toxic DPRs, poly-GA and poly-PR, respectively. We show that poly-PR expression caused seizures and ataxia in the absence of detectable neuronal loss in $40 \%$ of mice. In contrast, all poly-GA mice developed denervation of neuromuscular junctions and muscle wasting with eventual weight loss requiring euthanasia by $6-7$ weeks. We find selective vulnerability of the hippocampus and spinal motoneurons towards poly-GA.
Finally, we identify a C9orf72-enriched inflammatory signature shared between poly-GA mice and human C9orf72 ALS patients. The rapid progression of our new poly-GA mouse line and the strong inflammatory component that mimics the ALS spinal cord will allow rapid preclinical studies of poly-GA- and interferon-directed therapeutics.

\section{Materials and methods}

\section{Generation and breeding of mice}

We generated plasmids for conditional expression of DPRs by intersting GFP-GA 175 and GFP-PR 175 genes (encoded using non-repeating alternate codons) downstream of a floxed stop cassette (encoding a puromycin resistance gene followed by SV40 poly-adenylation signal) in the pEX CAG stop-bpA vector and electroporated them together with a PhiC31-Integrase expression plasmid into murine RMCE (recombinase-mediated cassette exchange) embryonic stem cells at the Rosa26 safe harbor [22]. The Rosa26 gene itself was knocked out by the neomycin-bpA gene trap during this process. Targeted integration was screened by neomycin selection and Southern blot. Clones with Creinducible DPR expression were injected into blastocytes. We identified mouse lines GAstop and PRstop with germline transmission and speed backcrossed them to C57BL/6N background until $>98 \%$ purity was confirmed using SNP genotyping (Charles River). Crossing GAstop and PRstop mice with Nestin-Cre [61] and subsequent excision of the stop cassette resulted in DPR expression throughout CNS neurons (ROSA26 GFP-GA ${ }_{175}{ }^{+/}$; Nestin-Cre and ROSA26 GFP-PR ${ }_{175}{ }^{+/-}$; Nestin-Cre). Relevant endpoint criteria for euthanasia according to national regulations were weight loss before 10 weeks of age or stage II seizures consisting of sustained cramping causing the mouse to fall on its side or back. Mice were killed by brief $\mathrm{CO}_{2}$ exposure, followed by decapitation. Tissue was either stored at $-80{ }^{\circ} \mathrm{C}$ for biochemistry analysis or formalin fixed for $24 \mathrm{~h}$ for immunohistochemistry analysis. For spinal cord tissue, decalcification using 5\% formic acid was performed for $48 \mathrm{~h}$.

Animal handling and animal experiments in this study were performed in accordance with the German animal welfare law and approved by the Government of Upper Bavaria, Germany. Mice were housed in standard cages in a pathogen-free facility in a 12-h light/dark cycle with ad libitum access to food and water.

\section{Rotarod}

14-Month-old animals were acclimated to the experimenter for 1 week before training by placing mice on the back of the hand and allowing them to explore for $1 \mathrm{~min}$. Training 
was performed on Monday, Wednesday and Friday, and animals were acclimated to the test room for $15 \mathrm{~min}$ before beginning. The apparatus was cleaned with $70 \% \mathrm{EtOH}$ and allowed to dry before each run. Mice were placed on the rotarod (RotaRod, TSE Systems) moving at $4 \mathrm{rpm}$ for three 1-min runs per day, each followed by a 10-min break. If mice fell off the rod, they waited at the bottom of the apparatus for the full minute. The following week, mice were tested for their latency to fall off the rotarod as it increased speed up to $60 \mathrm{rpm}$ over $1 \mathrm{~min}$. Each mouse again performed three testing trials each followed by 10 -min breaks.

\section{Immunohistochemistry and immunofluorescence}

Immunohistochemistry and immunofluorescence was performed on 5- $\mu \mathrm{m}$ paraffin-embedded tissue sections as described previously [37] using the following antibodies: Alyref (ab6141, Abcam 1:50), ChAT (AB144P, Millipore 1:200), GFP (632381, Takara 1:250), Fus (A300-292A, Bethyl Labs 1:100), Hnrnpa1 (sc-32301, Santa Cruz 1:50), Hnrnph (A300-511A, Bethyl Labs 1:500), Iba1 (091-19741, Wako, 1:500 and ab5076, Abcam, 1:500), Map2 (M1406, Sigma 1:250), NeuN (ab177487, Abcam, 1:1000), nucleolin (ab50279, Abcam 1:30), p62 (PM045, MBL 1:1000), polyGA (1A12, mouse, purified and biotinylated, 1:500) [37], poly-PR (32B3, mouse purified and biotinylated, Helmholtz Zentrum 1:1000) [58], Psmc4 (A303-850A, Bethyl Labs 1:250), Alexa Fluor 594 anti-Sfpq (A301-321A, Bethyl Labs 1:100), TDP-43N-terminal (10782-2-AP, Proteintech 1:500), TDP-43 p409/410 (1D3, gift from Manuela Neumann 1:500), TDP-43 p409/410 (CAC-TIP-PTD-P02, Cosmo 1:500), TDP-43 p409/410 (CAC-TIP-PTD-M01, Cosmo 1:1000), TDP43 p409/410 (3655, gift from Leonard Petrucelli 1:500), TDP-43 pS403/404 (CAC-TIP-PTDP05, Cosmo 1:500), TDP-43 pT153/Y155 (gift from Yuna Ayala 1:300), TDP-43 C-terminal (12892-1-AP, Proteintech 1:100), TDP-43 non-phospho 404-413 (TAR5 6D6, gift from Manuela Neumann 1:5), TDP-43 non-phospho 404-413 (TAR5 2H4, gift from Manuela Neumann 1:5), TDP-43 N-terminal (10782-2-AP, Proteintech 1:500), TDP43 p409/410 (1D3, gift from Manuela Neumann 1:500), TDP-43 p409/410 (CAC-TIP-PTD-P02, Cosmo 1:500), TDP-43 p409/410 (CAC-TIP-PTD-M01, Cosmo 1:1000), TDP43 p409/410 (3655, gift from Leonard Petrucelli 1:500), TDP-43 pS403/404 (CAC-TIP-PTD-P05, Cosmo 1:500), TDP-43 pT153/Y155 (gift from Yuna Ayala 1:300), TDP-43 C-terminal (12892-1-AP, Proteintech 1:100), TDP-43 nonphospho 404-413 (TAR5 6D6, gift from Manuela Neumann 1:5), TDP-43 non-phospho 404-413 (TAR5 2H4, gift from Manuela Neumann 1:5), Tubulin $\beta 3$ (801208, BioLegend 1:200). Nucleolin immunofluorescence required $1-5 \mathrm{~min}$ treatment with $50 \mu \mathrm{g} / \mathrm{mL}$ protease $\mathrm{K}$ prior to citrate antigen retrieval. Alexa Fluor 555 Mouse anti- $\beta$-Tubulin III (BD
Pharmingen 560,339 1:200) and Bungarotoxin Alexa Fluor 647 (Thermo Fisher B35450 1:500) were used to stain motor axons and acetyl-choline receptors at the NMJ, respectively.

\section{Immunofluorescence and histological quantifications}

Automated quantification of immunofluorescence was performed using CellProfiler (v. 3.0.0) software. For poly-PR quantification, images from sagittal sections approximately $100 \mu \mathrm{m}$ from the midline were taken covering the entire cortex of each animal. DAPI-stained nuclei and poly-PR aggregates were identified and counted, and the $\%$ of neurons with poly-PR inclusions was reported for each animal. For neuron quantification in brain and spinal cord, $10 \times$ scanning images were taken of the entire region on each slide (cortex, hippocampus, or spinal cord). NeuN-positive cells were identified using CellProfiler and divided by the area of the tissue section measured. Because the loss of NeuN can occur in intact neuronal nuclei [65], we also verified that there were no large nuclei with neuronal morphology in the CA fields of the hippocampus stained with DAPI but not NeuN. We used NeuN staining only to assist with semi-automated detection of neurons vs glia in our immunofluorescence staining. Choline acetyltransferase (ChAT)-positive cells with motor neuron morphology in the anterior horn were quantified manually on sections at 1-mm intervals spanning the whole spinal cord. For quantification of the denervated neuromuscular junctions (NMJs), confocal image stacks were recorded using an LSM810 confocal laser scanning system (Carl Zeiss) with $25 \times$ water immersion objective. Innervated or denervated single NMJs in the triangularis sterni and quadriceps muscles were classified by an investigator blinded to genotype.

\section{Western blotting and ELISA}

Tissues were collected on ice and lysed in 10 volumes of RIPA buffer with protease inhibitor and phosphatase inhibitor supplements. Tissue was centrifuged at $1000 \times g$ to remove cell debris but preserve DPR aggregates in the supernatant fraction. Protein concentration was determined by BCA assay. For western blot, $20 \mu \mathrm{g}$ of protein was loaded per lane on $10 \%$ SDS-acrylamide gels and separated by gel electrophoresis in Tris-glycine-SDS buffer, followed by transfer to PVDF membranes. Membranes were blocked with 5\% iBlock (Thermo Fisher, T2015) in TBS-Triton $\mathrm{x}-100$, or for detection of phosphorylated proteins in $5 \%$ bovine serum albumin (Thermo Fisher). We performed western blotting using the following antibodies: GFP (632381, Takara 1:1000), Stat1 (\#9172, Cell Signaling 1:1000), Tubb3 (TUJ1, Biolegend 1:1000), Dlg4 (PSD-95) (K28/74, Neuromab 1:1000), Syp (Sy38, Millipore 1:1000), Arc (sc-17839, 
Santa Cruz 1:200), pStat1 Tyr701 (\#7649, Cell Signaling 1:500), Stat2 (D9J7L, Cell Signaling 1:1k), pStat2 Y690 (ab53132, Abcam 1:500), Stat3 (\#79D7, Cell Signaling 1:1000), p-Stat3 Tyr705 (\#9145, Cell Signaling 1:1000), calnexin (SPA-860F, Enzo 1:7000). After washing in TBS$\mathrm{Tx}$, membranes were probed with anti-mouse or anti-rabbit HRP conjugated antibodies at 1:5000 in 5\% iBlock. HRPbound proteins were detected with ECL Western blotting substrate (Pierce, 32106). Cytokine ELISAs were performed according to the manufacturer's instructions using Legend Max mouse TNF $\alpha$ and IFN $\gamma$ ELISA kits (Biolegend) and read on a QuickPlex SQ 120 (Mesoscale).

\section{Hematological analysis}

Blood samples were collected by final blood withdrawal under isoflurane anesthesia by puncturing the retrobulbar vein plexus with a 1-mm-diameter glass capillary (Hirschmann). Blood was collected in EDTA-coated sample tubes (Kabe Labortechnik) and an amount of $50 \mu$ determined by an end-to-end capillary (Kabe Labortechnik) was diluted 1:5 with Cell Pack buffer (Sysmex) and stored at room temperature until analysis. To evaluate peripheral blood cell counts including automated differential white blood counts samples were mixed thoroughly by inverting the tube 3-4 times and analyzed using a Sysmex XT 2000iV hematology analyzer in the "capillary mode" with the predefined mouse settings within $6 \mathrm{~h}$ after sample collection and dilution.

\section{RNA isolation and library preparation}

Tissues were homogenized in Trizol using homogenizer (Precellys). Total RNA was isolated using Direct-zol-96 RNA isolation kit (ZymoResearch) following the manufacturer's instructions. RNA quantity and quality were controlled on Agilent 2100 BioAnalyzer using Agilent RNA 6000 Nano Kit.

Reverse transcription was performed at $42{ }^{\circ} \mathrm{C}$ for $90 \mathrm{~min}$ with a reaction consisting of 25 units Maxima $\mathrm{H}$ - enzyme (Thermo Fisher), $2 \times$ Maxima H-Buffer (Thermo Fisher), $2 \mathrm{mM}$ each dNTPs (Thermo Fisher), $4 \mu \mathrm{M}$ template-switching oligo-(IDT), $4 \mu \mathrm{M}$ barcoded oligo-dT primers (IDT), and $10 \mathrm{ng}$ of RNA, in a final reaction volume of $10 \mu \mathrm{L}$. Following RT, the cDNA was pooled and cleaned using SPRI beads, and then eluted with $17 \mu \mathrm{L}$ of DNase/RNase-Free Distilled Water (ThermoFisher). Residual primers were digested with Exonuclease I (Thermo Fisher) at $37{ }^{\circ} \mathrm{C}$ for $20 \mathrm{~min}$, with a subsequent heat inactivation step at $80{ }^{\circ} \mathrm{C}$ for $10 \mathrm{~min}$. Pre-amplification was then performed by adding $25 \mu \mathrm{L}$ of KAPA HiFi HotStart ReadyMix (2x, KAPABIOSYSTEMS) and $0.33 \mu \mathrm{M}$ SINGV6 primer (IDT) for a final reaction volume of $50 \mu \mathrm{L}$. The PCR was cycled as follows: $98^{\circ} \mathrm{C}$ for $3 \mathrm{~min}$ for initial denaturation followed by 11 cycles of $98^{\circ} \mathrm{C}$ for $15 \mathrm{~s}, 65^{\circ} \mathrm{C}$ for $30 \mathrm{~s}, 68^{\circ} \mathrm{C}$ for $4 \mathrm{~min}$. Final elongation was performed at $72{ }^{\circ} \mathrm{C}$ for $10 \mathrm{~min}$.

Following pre-amplification, the sample was cleaned using SPRI beads (1:0.8) and then eluted with $10 \mu \mathrm{L}$ of DNase/RNase-free distilled water (ThermoFisher). The cDNA was quantified using the Quant-iT PicoGreen dsDNA Assay Kit (Thermo Fisher) and size distributions were checked on high-sensitivity DNA chips (Agilent Bioanalyzer). After passing the quantity and quality controls, $0.8 \mathrm{ng}$ of pre-amplified cDNA was used to construct Nextera XT libraries (Illumina). The library PCR used custom P5 primer (P5NEXTPT5, IDT) to enrich for the $3^{\prime}$ ends. The library was then size selected using a $2 \%$ Agarose E-gel (Life Technologies) from 300-800 bp.

\section{qPCR}

qPCR was performed as described previously [69]. qRTPCR was performed using CFX384 Real-Time System (Bio-Rad) with Taqman technology. For GFP expression in the brain, primers and probes were designed (IDT) for the GFP region. Primer 1: GCACAAGCTGGAGTACAACTA, Primer 2: TGTTGTGGCGGATCTTGAA, Probe/56FAM/ AGCAGAAGA/ZEN/ACGGCATCAAGGTGA/3IABkFQ/. Signals were normalized to GAPDH levels according to $\Delta \Delta \mathrm{CT}$ method and levels are shown relative to control. For GFP expression in the muscle, Taqman probes (ThermoFisher) were used against GFP (Mr04097229_mr) and Atp5a1 (Mm00431960_m1) with Sybr fluor. GFP signals were normalized to Atp5a according to the $\Delta \Delta \mathrm{CT}$ method and levels are shown relative to control.

\section{Sequencing and primary data processing}

Final libraries were paired-end sequenced on a high output flow cell of a HiSeq 1500 (Illumina), with the following sequencing setup: 16 bases for the cellular barcode and UMI, eight bases for the i7 barcode, and 50 bases for the cDNA read. Libraries were sequenced to a depth of $\sim 10$ million reads per sample. Raw fastq files were checked using FastQC (v 0.10.1) and then processed using zUMIs (v 0.0.6) with default settings and a provided barcode list. The data were mapped to the mouse genome $(\mathrm{mm} 10)$ and gene annotations were obtained from Ensembl (GRCh38.89).

\section{Data analysis}

The count tables (from tissue mean counts $2.4 \mathrm{M}$, range 0.225-28 M; from isolated microglia average counts $250 \mathrm{~K}$, range 153-678 K; samples with less than $100 \mathrm{~K}$ counts were excluded) were analyzed using the DESeq2 pipeline in $\mathrm{R}$ comparing transgenic mice and littermate 
controls for each region and age separately [36]. The genotype of all mice was confirmed (and corrected) using the GFP-(GA) 175 and GFP-(PR) 175 transcript. The number of samples per group was 3 weeks ( 7 control vs 3 GA-Nes), end-stage ( 5 control vs 5 GA-Nes), isolated microglia at end-stage ( 3 control vs 3 GA-Nes), affected ( 5 control vs 5 PR-Nes), and asymptomatic (3 control vs 5 PR-Nes). All significant hits $(p<0.05$ adjusted for multiple testing for each pairwise comparison) are listed in Table S2. All publicly available RNAseq data from TargetALS data were accessed in February 2020. We included all samples from patients with ALS and controls without CNS comorbidity and with $C 9$ orf 72 -genotype information available. Reads were aligned to GRCh38 using STAR aligner b2.4.2a [12] by the New York Genome Center and further processed by us using SummarizeOverlaps in R. Count tables were analyzed using DESeq2 [36] in a linear model accounting for gender as a confounder in the cerebellum (14 controls, 108 ALS, and 30 C9orf72 ALS) and frontal cortex (16 controls, 90 ALS, and 26 C9orf 72 ALS), and differential gene expression was analyzed between the relevant groups. A model accounting for gender and anatomical sub-regions was used for spinal cord (cervical: 13 controls, 86 ALS, 25 C9orf72 ALS; thoracic: eight controls, 44 ALS, 7 C9orf72; lumbar: 11 controls, 77 ALS, 19 C9orf72 ALS) and motor cortex (lateral: 13 controls, 77 ALS, 21 C9orf72 ALS; medial: 15 control, 78 ALS and 18 C9orf72 ALS). The overall LFC for the groups was used for the correlation analysis.

Gene ontology analysis (biological process) was done using the clusterProfiler package in R. Only groups with more than five significant genes were analyzed. Figures show a manual selection of the most significant and least overlapping categories to best represent the key pathways. The full list of significant GO terms is shown in Tables S3 and S5. Concordantly regulated genes were defined by comparing averaged significant up-/down-regulation from all analyzed regions in GA-Nes or PR-Nes mice and ALS patients.

Kmeans clustering was done using default parameters with pheatmap (1.0.12) in R. Pearson's correlation of gene expression was done using pairwise-complete observations with identical Entrez gene names. We used the $\log 2$-fold expression changes of significant genes from the AD and SOD1 mouse models (padj <0.05) [26], and the p25 mouse model in reference [40] $(\mathrm{cZ}>3.0902)$. For the microglia clusters, we used all genes listed in the supplemental data of reference [19]. The actual number of genes used is indicated in the figure legends.

\section{Statistics}

Statistical analyses other than for RNAseq data (described separately above) were performed using GraphPad Prism 7.02. We used two-tailed $t$ tests to compare two groups, and ANOVAs with Greenhouse-Geisser correction to compare more than two groups. Data are reported in the figure legends with (mean \pm standard deviation) and effect size $\left(\eta^{2}\right.$, the between-group sum-of-squares divided by the total sum-of-squares). Confidence intervals are reported instead of effect size for non-parametric tests. Data distributions were checked for normality by the Shapiro-Wilk test and homogeneity of variances was checked by the $F$ test or the Brown-Forsythe test. When these were violated, non-parametric tests were used (Mann-Whitney). Individual points for immunoblots represent the average of at least two independent replicates per biological sample. Power analyses were performed with GPower 3.1.

\section{Results}

\section{Poly-GA expression causes progressive weakness, while poly-PR triggers ataxia and seizures with high expression and no symptoms with low expression}

To assess only the contribution of the DPR proteins in the absence of $\mathrm{G}_{4} \mathrm{C}_{2}$ RNA toxicity, we used non-repeating alternate codons to individually express poly-GA and poly-PR in the absence of $\left(\mathrm{G}_{4} \mathrm{C}_{2}\right)_{n}$ repeats as in previous models [41, 57]. We generated conditional single transgenic DPR expressing mice by targeted insertion of a GFP-GA 175 or GFP-PR 175 expression construct driven by the strong CAG promoter with a LoxP-flanked stop cassette at the Rosa26 safe harbor locus (Fig. S1a). To allow CNS-wide neuronal expression we crossed these mice with the well-characterized Nestin-Cre driver line (Fig. S1a, b) [61] creating double transgenic mice (GA-Nes and PR-Nes). GA-Nes and PRNes mice were born at Mendelian ratios $\left(23.5 \% \chi^{2}=2.327\right.$, $d f=3, p=0.5075 n \geq 44 /$ group and $23.5 \% \chi^{2}=6.647, d f=3$, $p=0.084 n \geq 24$ /group, respectively).

We observed progressive motor deficits and early lethality in all GA-Nes mice and a subset of PR-Nes mice. All GA-Nes mice showed abnormal gait and impaired motor function from 5 weeks (S. video 1 ), and by 6-7 weeks mice had severe muscle weakness but not paralysis (S. video 2). GA-Nes mice were smaller than control littermates beginning around 4 weeks of age, and later even began to lose weight, at which time we were required to euthanize them in accordance with national animal welfare laws. All GA-Nes mice reached this end-stage point before 7 weeks, resulting in a sharp drop in the Kaplan-Meier curve (Fig. 1a), and providing food on the floor of the cage did not improve survival. 
a
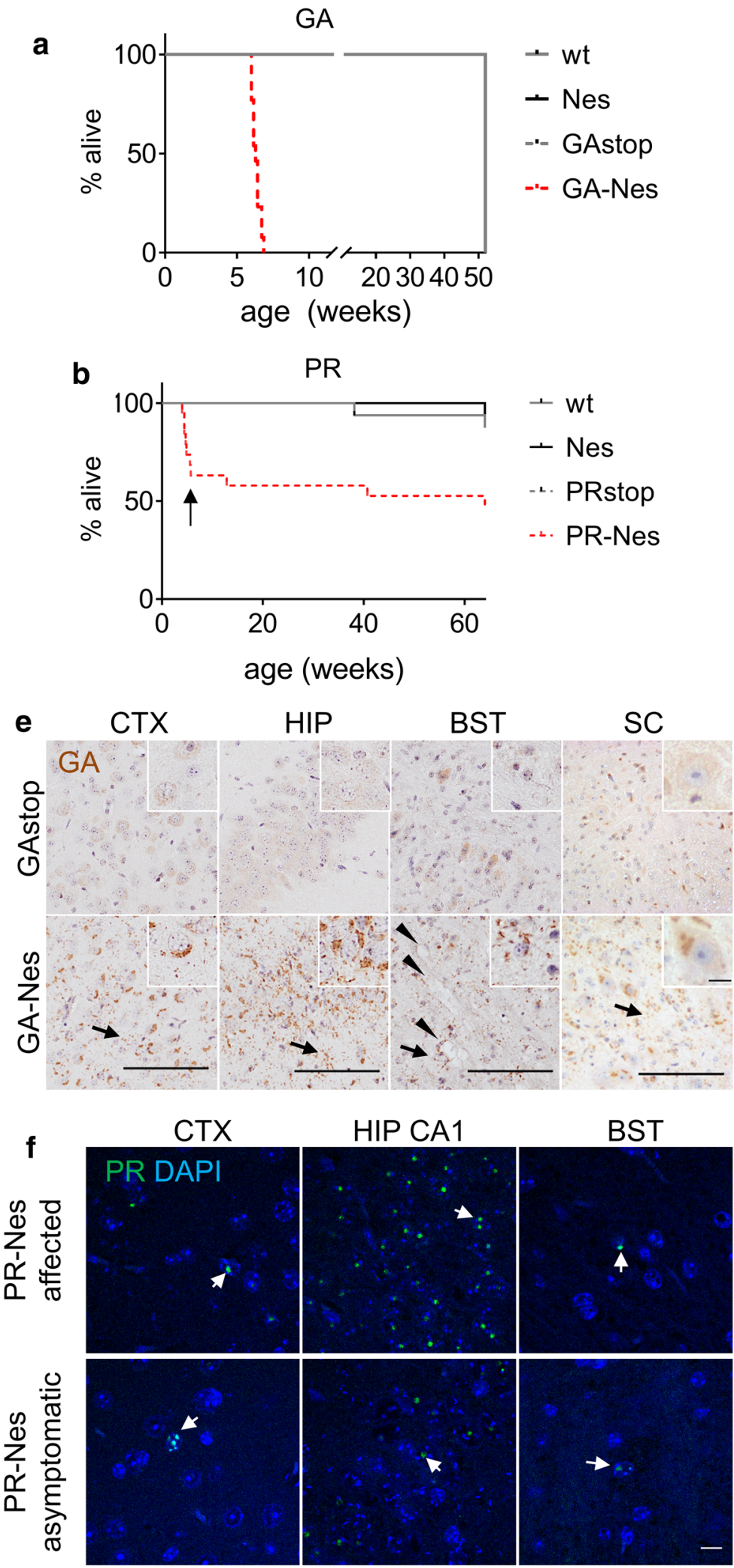

- wt

- Nes

-- GAstop

-"- GA-Nes

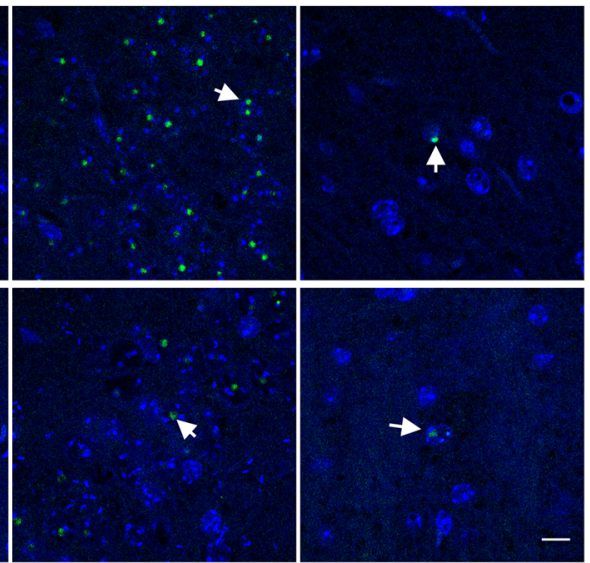

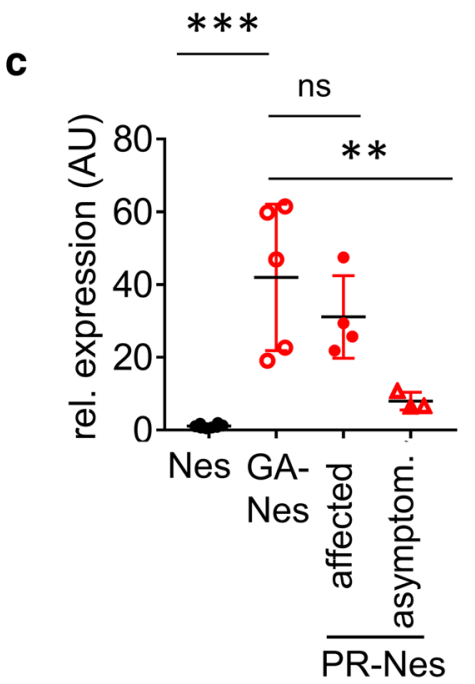

d

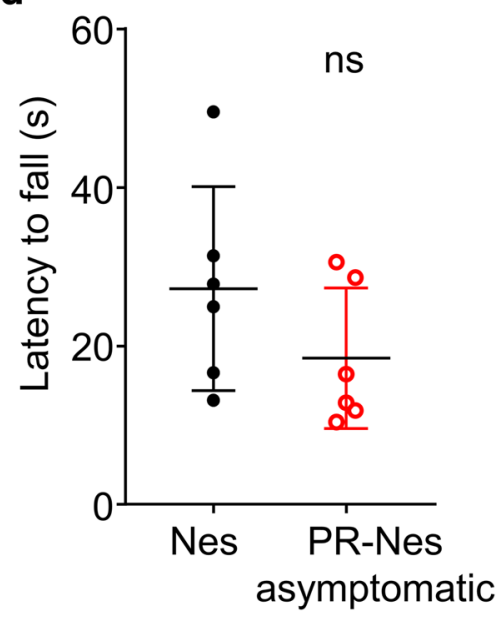

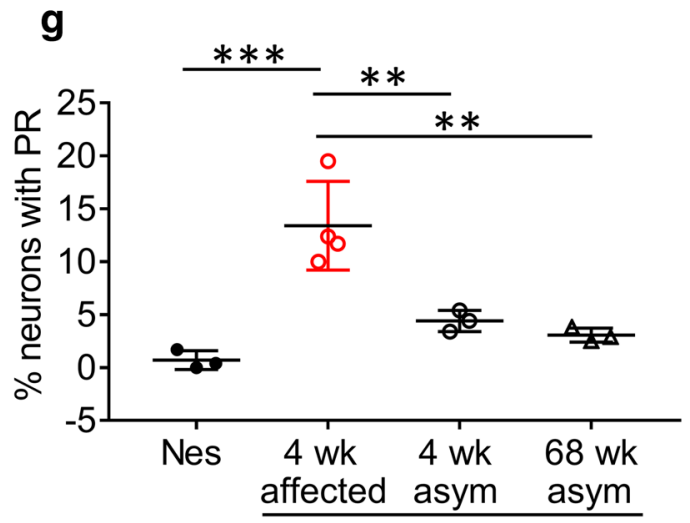

PR-Nes

In contrast, about $40 \%$ of PR-Nes mice also failed to thrive and around 4-5 weeks of age developed seizures that were criteria for euthanasia (Fig. 1b). These mice also showed poor coordination reminiscent of ataxia and fell frequently to the side without apparent muscle weakness (Supp. video 3). The remaining PR-Nes mice that did not meet endpoint 
४Fig. 1 All GA-Nes mice and a subset of PR-Nes mice reach the humane endpoint rapidly, while remaining PR-Nes mice downregulate transgene expression and live normal life spans without motor deficits. a All GA-Nes mice reach the humane endpoint by 7 weeks of age due to weakness, while all animals of control genotypes remain healthy until the end of the study at 52.1 weeks. Mantel-Cox $\chi^{2}=44.35, p<0.001$. Median endpoint $\mathrm{wt}=52.1 \quad(n=9), \mathrm{Nes}=52.1$ $(n=5)$, GAstop $=52.1(n=8)$, GA-Nes $=6.3(n=13)$. b $40 \%$ of PRNes mice reach the humane endpoint by 5 weeks of age due to seizure and ataxia and make up the affected group (arrow). The other mice remain asymptomatic. Mantel-Cox $\chi^{2}=11.32, p=0.01$. Median endpoint $\mathrm{wt}=69.9(n=16), \mathrm{Nes}=70(n=16)$, PRstop $=70.1(n=11)$, PR-Nes $=69.3(n=19)$. Study duration: 68 weeks. c Expression of poly-GA and poly-PR mRNA is comparable between GA-Nes and affected PR-Nes mice, but not asymptomatic PR-Nes (AU =arbitrary units).[ANOVA $\mathrm{F}(3,16)=16.2 p<0.001 \eta^{2}=0.753$, Dunn's post hoc Nes $(1.1 \pm 0.51)$ vs GA-Nes $(42 \pm 20.1) * * * p<0.001$, GANes $(42 \pm 20.1)$ vs affected PR-Nes $(31.1 \pm 11.3)$ ns $p=0.365$, GANes(42 \pm 20.1$)$ vs asymptomatic PR-Nes $(8.0 \pm 2.4) * * p=0.002]$. Nes $n=8$, GA-Nes $n=5$, affected PR-Nes $n=4$, asymptomatic PR-Nes $n=3$. d Rotarod assay shows no significant difference in latency to fall $(\mathrm{s}=$ seconds) at 14 months of age $[t(6)=1374$, Nes $(27.2 \pm 12.9)$ vs asymptomatic PR-Nes $\left.(18.43 \pm 8.9) \quad p=0.199 \quad \eta^{2}=0.159\right]$ $n=6$ per group. Power to detect $\eta^{2}>0.5=52 \%$. e Representative images of GA-GFP immunohistochemistry staining (brown) at 3 weeks in cortex (CTX), hippocampus CA2 (HIP), brainstem (BST) and spinal cord (SC), including motor neurons (inset shown). PolyGA aggregates are also frequent in the neuropil (arrows). Counterstain hematoxylin. Extracellular vacuoles are visible near axon tracts in the brainstem (arrowheads). Scale bar $=100 \mu \mathrm{m}$, insets $=10 \mu \mathrm{m}$. f Representative images of PR-GFP immunofluorescence shows predominantly low-abundance nuclear aggregates (arrows) in the neocortex (CTX) and brainstem (BST), but higher abundance in the CA1 region of the hippocampus (HIP CA1), especially in affected mice, scale bar $=10 \mu \mathrm{m}$. For visibility in printed versions, the intensity of the green channel was doubled in all images. Closer views of PR aggregates are shown in Fig. 2i,j. g Automated quantification of the $\%$ of neurons with poly-PR inclusions in the neocortex of affected and asymptomatic (asym) PR-Nes and control (Nes) mice [ANOVA $\mathrm{F}(3,9)=17.7, p<0.001 \eta^{2}=0.855$; Holm-Sidak post hoc $\operatorname{Nes}(0.7 \pm 0.889)$ vs 4 weeks affected $(13.4 \pm 4.2) * * * p=0.001,4$ weeks affected $(13.4 \pm 4.2)$ vs 4 weeks asym $(4.4 \pm 1.0) * * p=0.005$, 4 weeks affected $(13.4 \pm 4.2)$ vs 68 weeks asym $(3.1 \pm 0.7)$ ** $p=0.002]$. 4 weeks Nes $n=3,4$ wk affected $n=4,4$ weeks asym $n=3,68$ weeks asym $n=3$

criteria early on did not develop seizures later in life, gained weight normally and had normal motor function until the end of the study at 68 weeks (Supp. video 4). The rotarod assay revealed no significant motor deficits in these mice even at 14 months (Fig. 1d). Due to these radically different phenotypes, also seen to a lesser extent upon viral poly-PR expression [68], we separated PR-Nes mice into affected (4 weeks) and asymptomatic (4 weeks and 68 weeks) groups for the remaining studies. qPCR analysis showed similar transgene expression at the mRNA levels in the cortex of GA-Nes mice and affected PR-Nes mice, but significantly reduced transgene expression in asymptomatic PR-Nes mice (Fig. 1c). Genomic PCR confirmed that asymptomatic PRNes still maintained the 175-repeat length suggesting transcriptional repression of the synthetic construct (Fig. S1c).
To elucidate the differential phenotypes in GA-Nes and PR-Nes mice, we first compared DPR expression patterns in both lines using immunostaining. As expected, in the absence of Cre expression (GAstop), no poly-GA expression was detected (Fig. 1e). Consistent with the pan-neuronal expression of the Nestin-Cre driver, poly-GA formed frequent neuronal cytoplasmic inclusions (NCI) and neuritic aggregates in the vast majority of neurons throughout the CNS of GA-Nes mice (Fig. 1e). Combined fluorescent in situ hybridization (FISH) to label the transgene mRNA and immunostaining of Cnp1, GFAP and Iba1 revealed transgene expression in a subset of oligodendrocytes and astrocytes, but not in microglia (Fig. S1d-f). Importantly, despite similar mRNA levels in GA-Nes and affected PRNes mice, poly-PR inclusions were infrequent in the cortex and brainstem (Fig. 1f) and not found in the spinal cord. However, in the hippocampus, most neurons had at least one nuclear inclusion in affected PR-Nes mice, while far fewer hippocampal neurons contained inclusions in asymptomatic PR-Nes mice (68 weeks) (Fig. 1f). Quantification of poly-PR in the cortex showed that inclusions in asymptomatic PRNes mice were already reduced at 4 weeks, and this persisted in asymptomatics at 68 weeks (Fig. 1g). By immunoblotting with a GFP antibody, poly-PR (aggregated or soluble) was below the detection level even in affected mice, in contrast to abundant aggregated poly-GA visible in the gel pocket (Fig. S1g). Poly-PR aggregates were barely detectable when 25 times more brain extracts than necessary for poly-GA detection were loaded on a filter trap assay (Fig. S1h). Our findings are consistent with the abundant poly-GA and scarce poly-PR inclusion pathology observed in C9orf72 patients $[38,58]$, though both are more frequent than observed in patients. Overall, the comparison between these congenic lines shows consistent progressive weakness in poly-GA mice that more closely resembles ALS than the ataxia and seizures found in poly-PR mice, a subset of which escape toxicity completely by reducing transgene expression.

\section{Selective neuron loss and microglial infiltration in the hippocampus of poly-GA but not poly-PR mice}

To address the consequences of DPR expression and identify correlates of the motor deficits, we analyzed regional neuron loss and early signs of neurodegeneration and microglial activation in our mouse models. Despite widespread aggregation of poly-GA in the vast majority of CNS neurons, robust microglial infiltration accompanied by mild neuron loss first became apparent in the CA2 of the hippocampus after 3 weeks of age, with nearly complete ablation of the CA2 and thinning of the other CA fields by end-stage (Fig. 2a, b; Fig. S2a). The CA2 is delineated by the end of the compactly layered neurons of the CA1 and the beginning of the larger and more diffusely spaced pyramidal neurons 

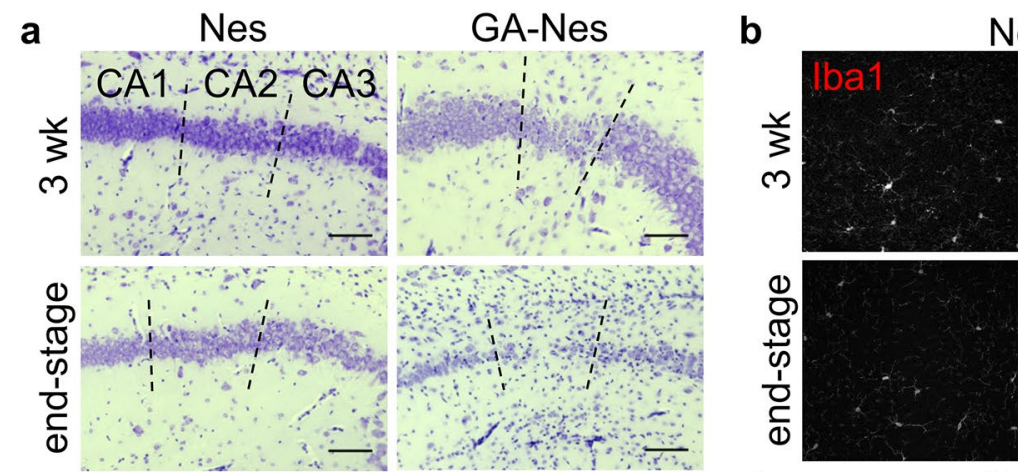

Nes

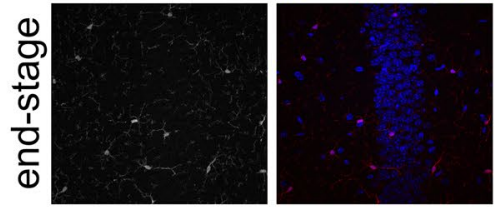

c

Nes

PR-Nes

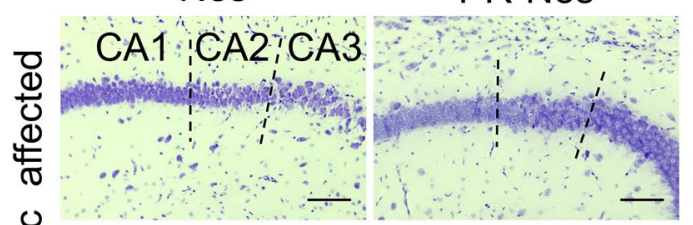

d

Nes
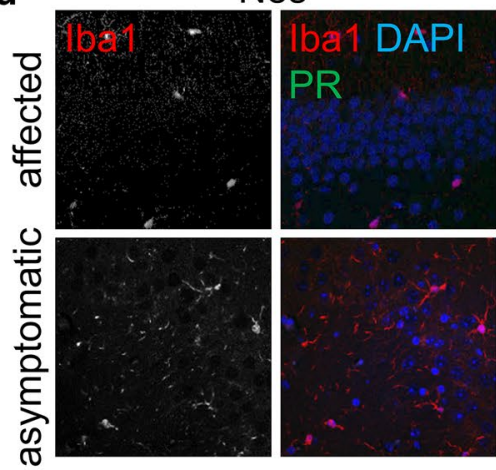

Hip CA1-3

GA-Nes

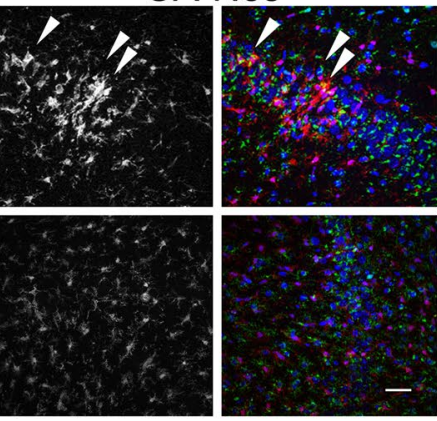

PR-Nes

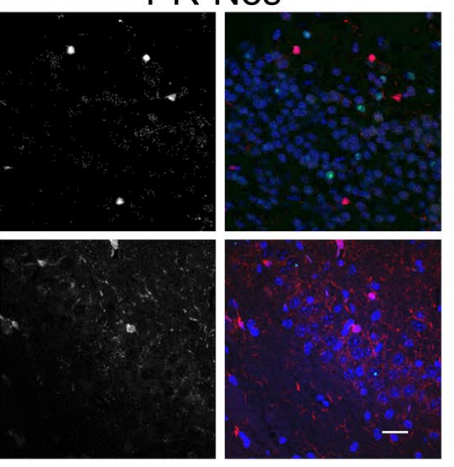

e Hip CA1-3 Cortex L2-6

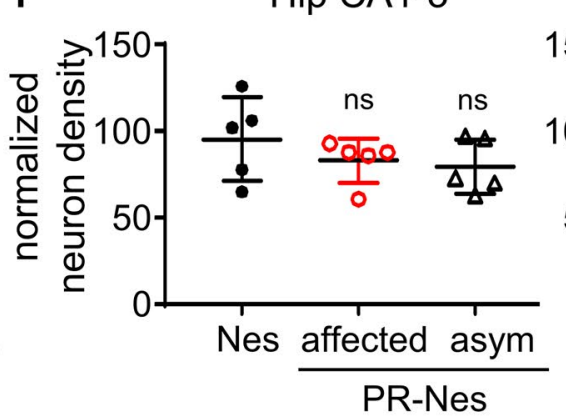

Cortex L2-6

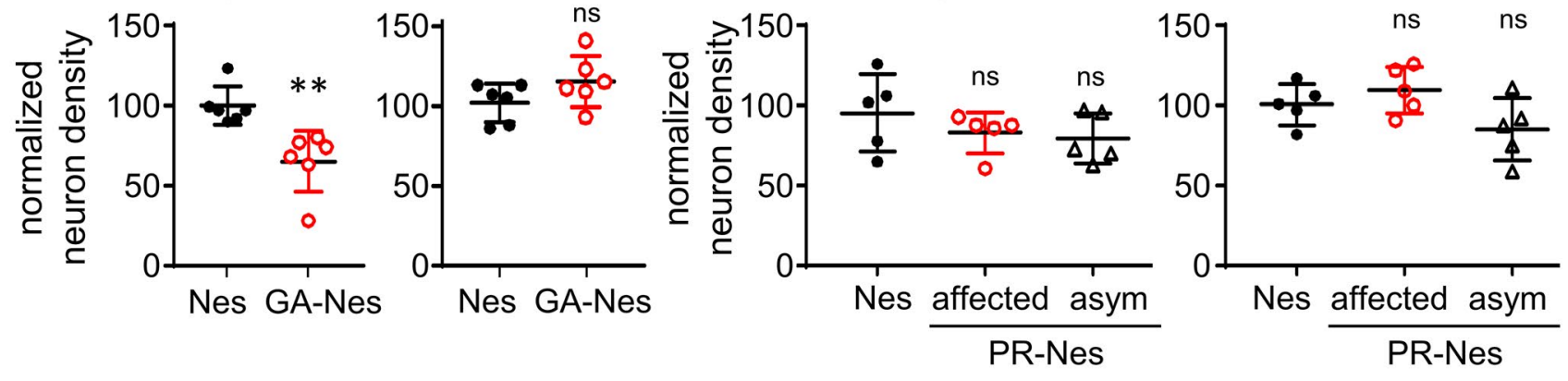

g

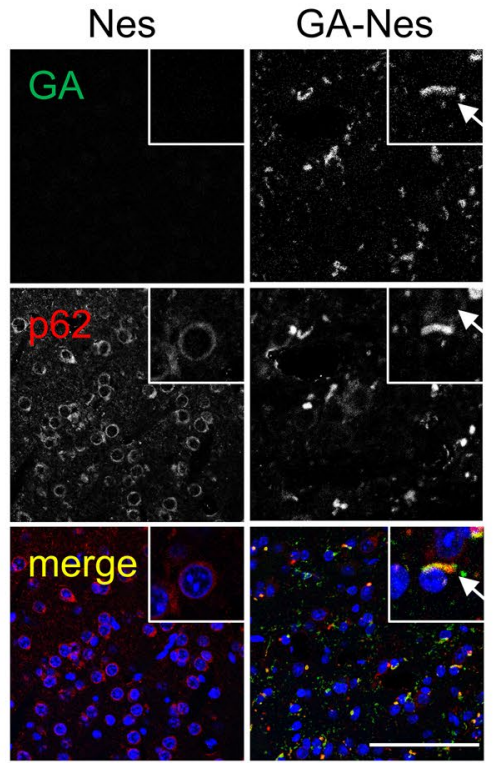

h

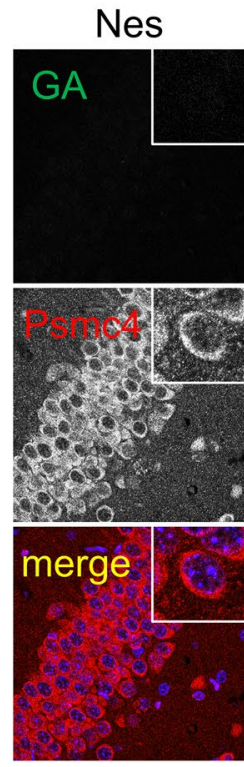

GA-Nes

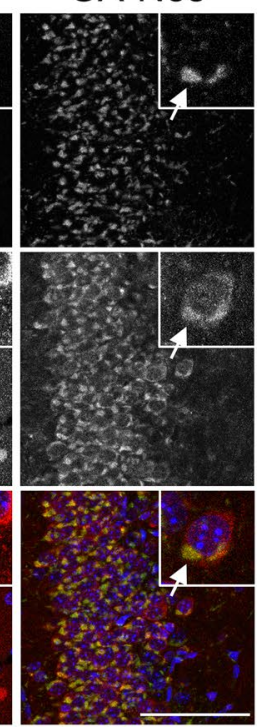

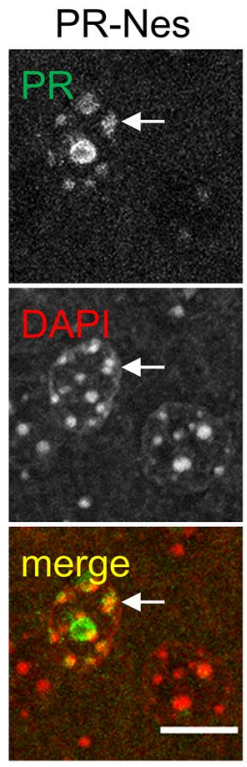

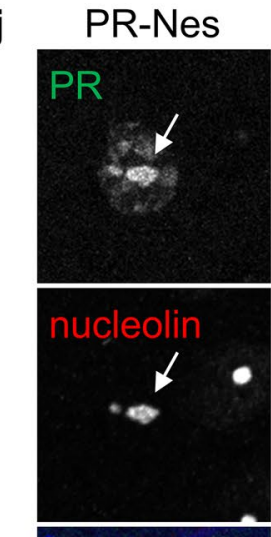

merge DAPI

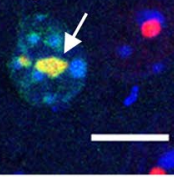


4Fig. 2 Poly-GA leads to early microglial infiltration in the hippocampus preceding neuron loss, while poly-PR does not. a Nissl stains show thinning of the CA neuronal layers in GA-Nes mice beginning at 3 weeks, causing nearly complete ablation of the CA2 by endstage in contrast to Nes control mice, scale bar $=100 \mu \mathrm{m}$. For wider views see Fig. S2a. b Clusters of activated amoeboid microglia (Iba1, red) infiltrate the CA2 in GA-Nes mice at 3 weeks of age, despite GA aggregates (GFP, green) in virtually all surrounding neurons. Microglia return to ramified morphology and are homogeneously distributed in the CA2 and the surrounding region by end-stage, scale bar $=25 \mu \mathrm{m}$. $\mathbf{c}$ Nissl stains show no thinning of the CA neuronal layers in affected or asymptomatic PR-Nes mice (right column) compared to Nes controls (left column), scale bar $=100 \mu \mathrm{m}$. For wider views see Fig. S2b. d Immunofluorescence shows normal ramification and no clustering of microglia (Iba1) in the hippocampus of PRNes affected or asymptomatic mice compared to Nes controls despite the presence of PR aggregates (GFP, green), scale bar $=25 \mu \mathrm{m}$. e GA-Nes mice show significant neuron loss (NeuN positivecells) in the CA1-3 of the hippocampus [Welch's $t(8.37)=3.77, * * p=0.005$ $\eta^{2}=0.629$, Nes $(99.7 \pm 11.9)$ GA-Nes $\left.(65 \pm 19.1)\right]$, but not in the neocortical layers (L2-6) $\left[t\right.$ test $t(10)=1.63$, ns $p=0.135, \eta^{2}=0.21$, Nes $(102 \pm 12.1)$ GA-Nes(115 \pm 16$)] . n=6 /$ group. f No neuron loss (NeuN positive cells) in the hippocampus or neocortex of affected or asymptomatic (asym) PR-Nes mice [hippocampus: Kruskal-Wallis $=1.82, p=0.427$, Nes $(95.4 \pm 24.1$, CI 65.5-125) affected PR-Nes (83.2 \pm 12.7 , CI 67.5-98.9) asymptomatic PR-Nes (79.8 \pm 15.7 , CI 60.3-99.3); neocortex: ANOVA $F(2,12)=0.307, p=0.084 \eta^{2}=0.338$, Nes $(101 \pm 12.8)$ affected PR-Nes $(110 \pm 14.7)$ asymptomatic PR-Nes $(85 \pm 19.4)] . n=5$ /group. $g$ Double immunofluorescence shows colocalization (arrows) of poly-GA aggregates (GFP) with p62 (Sqstm1) in the neocortex (scale bar $=100 \mu \mathrm{m})$. Insets $(30 \times 30 \mu \mathrm{m})$ provide a close view of single neurons. DAPI (blue). h Double immunofluorescence shows colocalization (arrows) of poly-GA aggregates (GFP) and Psmc4 in the CA1 of the hippocampus at 3 weeks (scale bar $=100 \mu \mathrm{m})$. Insets $(30 \times 30 \mu \mathrm{m})$ provide a close view of single neurons. DAPI (blue). i Double immunofluorescence shows colocalization (arrows) of poly-PR (GFP, green) and heterochromatin (DAPI, red) in the neocortex of an affected PR-Nes mouse. This colocalization was also observed in asymptomatic mice. Scale bar $=10 \mu \mathrm{m} . \mathrm{j}$ ) Representative images of the colocalization (arrows) of PR (GFP, green) and nucleolin (red) in the neocortex of an asymptomatic PRNes mouse. This colocalization was also observed in affected mice. DAPI (blue) shows the area of the nucleus. Scale bar $=10 \mu \mathrm{m}$

of the CA3. In contrast, both affected and asymptomatic PR-Nes mice showed normal neuron density (Fig. 2c, f, Fig. S2b) and no microglia activation in the hippocampus (Fig. 2d), despite high poly-PR aggregation there (compare Fig. 1f). Surprisingly, infiltration of activated microglia with typical amoeboid morphology in the CA2 region of GANes mice was stronger at 3 weeks of age than at end-stage (Fig. 2b). Quantification at end-stage confirmed significant neuron loss in the whole CA field, even when normalizing to the area of the region in the overall smaller brains (Fig. 2e, Fig. S2a). In contrast, neuron loss was not detected in the cortex of GA-Nes (Fig. 2e, Fig. S2a) or affected PR-Nes mice (Fig. 2f, Fig. S2b).

To address known toxicity mechanisms, we analyzed the subcellular distribution of poly-GA and poly-PR in our mice. Poly-GA co-aggregated with p62/Sqstm1 (Fig. 2g) and the proteasome subunit Psmc4 (Fig. 2h), consistent with cryo-electron tomography data in primary neurons [18]. In PR-Nes mice, poly-PR was restricted to the nucleus and colocalized with heterochromatin (Fig. 2i) and the nucleolus (Fig. $2 \mathrm{j}$ ) in both affected and asymptomatic mice, as reported in other models [21, 68]. However, despite subcellular localization of both DPRs matching that of other mouse models, only poly-GA led to detectable neuron loss in the hippocampus.

\section{Selective motoneuron loss and muscle denervation in GA-Nes but not PR-Nes mice}

To investigate the muscle weakness in GA-Nes mice, we also examined spinal cord neurons and neuromuscular junctions. While ChAT-positive motor neurons in the anterior horn of the spinal cord were normally abundant in GA-Nes mice at 3 weeks, they were significantly lost by end-stage (Fig. 3a, b). In contrast, the number of calbindin-positive neurons in the dorsal horn was unchanged even at end-stage (Fig. S2c, d), suggesting selective vulnerability of motor neurons in the spinal cord to poly-GA. Consistent with the lack of muscle wasting in PR-Nes mice, no loss of ChAT-positive neurons in the spinal cord was observed (Fig. 3c, d). We further investigated axonal innervation of neuromuscular junctions in GA-Nes mice. While neuromuscular junctions were fully innervated at 3 weeks in GA-Nes mice, by end-stage most $\alpha$-bungarotoxin-labeled endplates were denervated, as indicated by loss of $\beta$-tubulin III immunostaining in both triangularis sterni (whole-mount preparation $[4,27]$ ) and quadriceps muscles (vibratome sections) (Fig. 3e, f). In contrast, no denervation was observed in the triangularis sterni muscle of PR-Nes affected or asymptomatic mice (Fig. 3g).

Despite the clear correlation between motoneuron loss and weakness, we investigated possible peripheral causes of muscle wasting requiring early euthanasia. Hematological parameters, including white blood cell counts, were not significantly different between GA-Nes and controls (Fig. S3a). Systematic macroscopic and microscopic analyses were conducted in the intestines, pancreas, liver, kidney, adrenal gland, spleen, heart, lung, and thymus. Consistent with the known expression profile of Nestin-Cre [11, 13], GFP staining revealed sparse expression of poly-GA in the kidney and pancreas, as well as in neurons of the myenteric plexus (Fig. $\mathrm{S} 4 \mathrm{a})$. However, $\mathrm{H}+\mathrm{E}$ staining revealed no signs of necrosis or gross pathological abnormality in these or other organs compared to control littermates, other than smaller size consistent with the overall smaller size of GA-Nes mice (Fig. $\mathrm{S} 4 \mathrm{~b}$ ). In addition, the examination of the intact digestive tract at euthanasia showed processed boli present at regular intervals throughout the entire digestive tract in both GANes and controls arguing against impaired peristalsis or intestinal transit (Fig. S4c). Poly-GA aggregates were also found commonly in the quadriceps muscle (Fig. S4d), again 
a

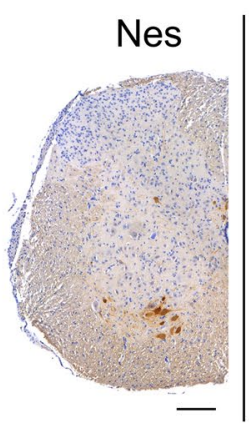

GA-Nes

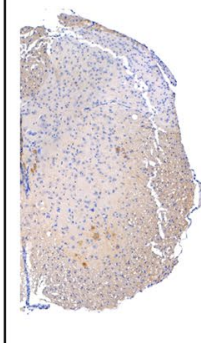

b

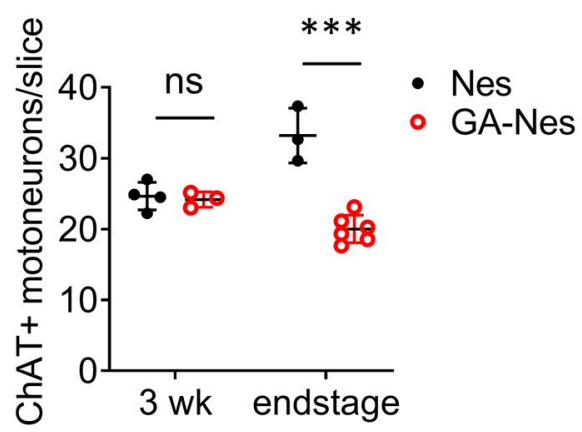

C
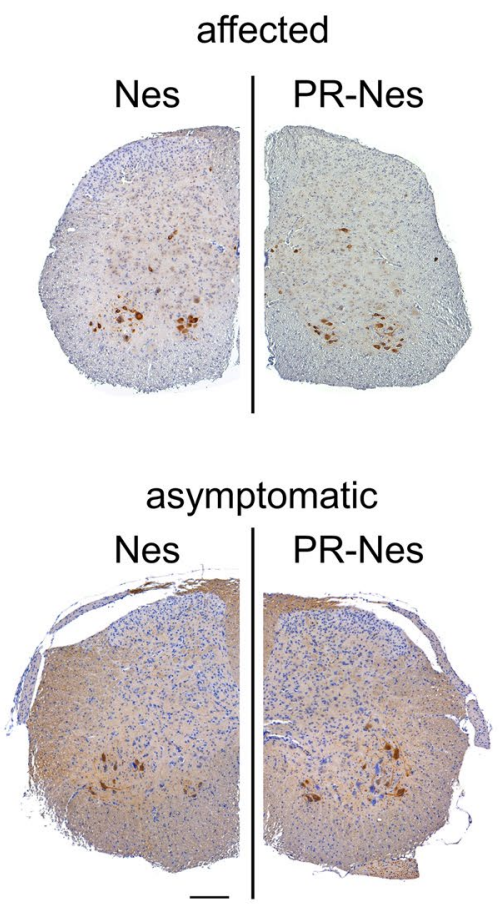

d

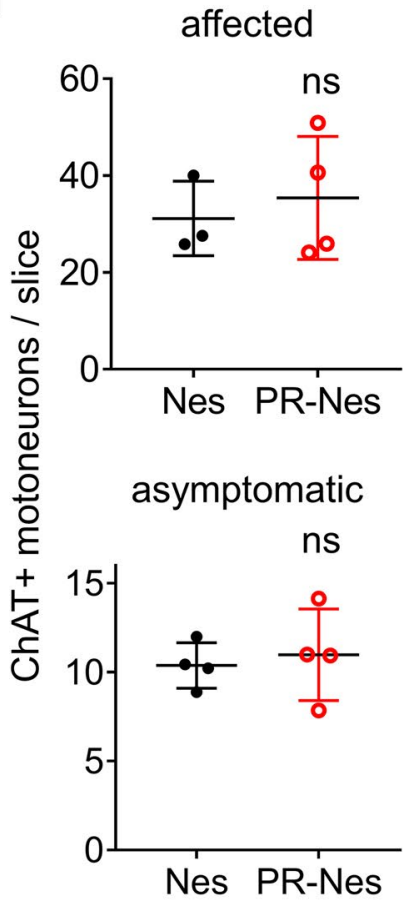

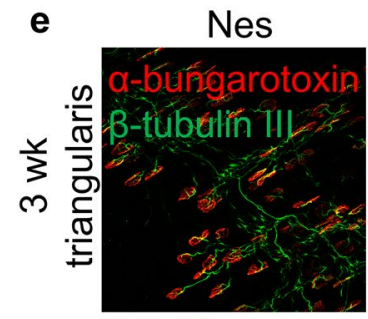
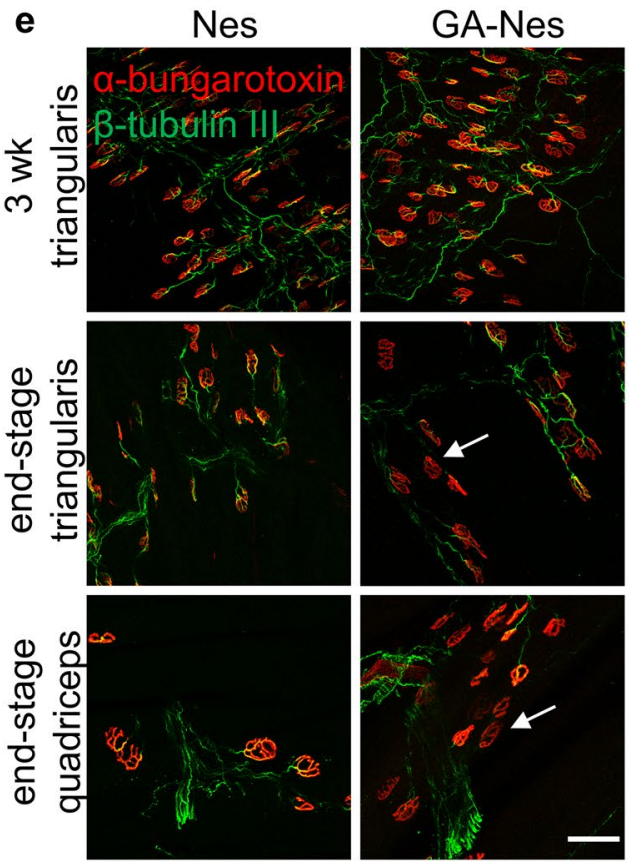

f
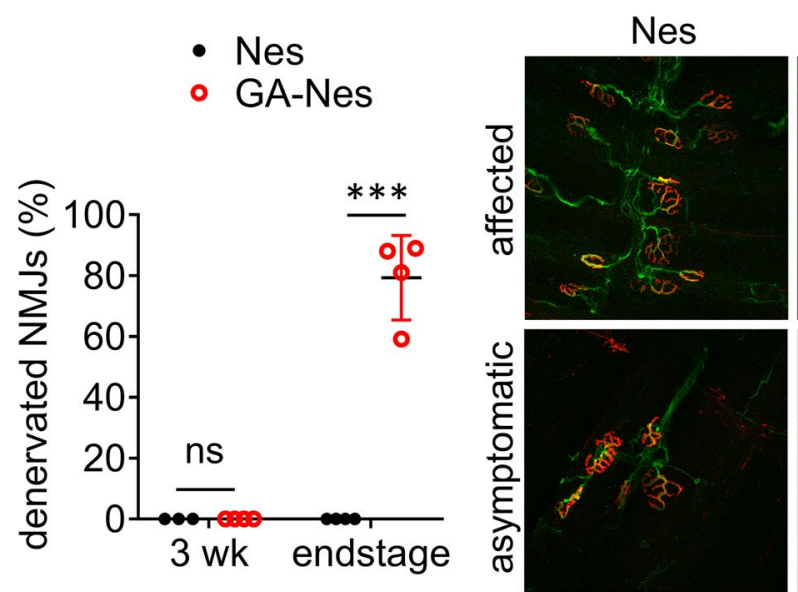

PR-Nes

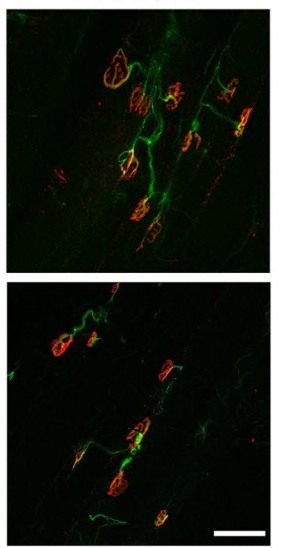

consistent with known expression of Nestin-Cre [13] and with the muscle wasting and denervation observed. FISH and qPCR analysis of the quadriceps confirmed that polyGA was expressed locally in skeletal muscle (Fig. S4e, $\mathrm{f}$ ), which is particularly interesting given recent observations that poly-GA is commonly found there in human C9orf72 ALS cases [9]. Thus, selective loss of motoneurons and neuromuscular denervation together with potential cell-autonomous effects in the muscle are likely the predominant drivers of progressive muscle wasting in GA-Nes mice.

\section{TDP-43 and other disease-linked RNA-binding proteins form nuclear inclusions in GA-Nes mice}

To elucidate the elusive link between DPR and TDP-43 pathology, we characterized TDP-43 localization in GA-Nes and PR-Nes mice in detail using a panel of antibodies (Fig 
४Fig. 3 End-stage GA-Nes mice develop selective neurodegeneration in hippocampus and spinal cord and muscle denervation, while PRNes mice do not. a Immunohistochemical visualization of motoneurons by antibodies against ChAT (brown) in the lumbar spinal cord shows a clear reduction of ChAT expressing cells in the end-stage GA-Nes mouse compared to Nes control. Scale bar $=200 \mu \mathrm{m}$. b Quantification of average ChAT + motoneurons/slice sampled every $1 \mathrm{~mm}$ through the whole spinal cord. GA-Nes mice show significant motor neuron loss by end-stage compared to Nes controls. [Twoway ANOVA interaction (age $\mathrm{x}$ genotype) $\mathrm{F}(1,12)=28.6, p<0.001$ $\eta^{2}=0.705 ; 3$ wk Nes $(24.7 \pm 2.0, n=4)$ vs GA-Nes $(24.2 \pm 1.1, n=3)$ ns $p=0.96$; end-stage Nes $(33.2 \pm 3.9, n=3)$ vs GA-Nes $(20.1 \pm 2.0$, $n=6) * * * p<0.001$.] c ChAT immunohistochemistry (brown) visualizing motoneurons in the lumbar spinal cord of PR-Nes affected and asymptomatic mice and age-matched Nes mice shows no obvious differences in the number of ChAT expressing cells among all mice. Scale bar $=200 \mu \mathrm{m}$. d Quantification of average ChAT + motoneurons/slice sampled every $1 \mathrm{~mm}$ through the whole spinal cord of affected and asymptomatic PR-Nes mice and age-matched Nes controls shows no significant motor neuron loss in either PR-Nes group. Nes $(31.1 \pm 7.7, n=3)$ vs affected PR-Nes $(35.4 \pm 12.7$, $n=4)\left[t(5)=0.505, p=0.635 \eta^{2}=0.0485\right]$. Nes $(10.4 \pm 1.3, n=4)$ vs asymptomatic PR-Nes $(11 \pm 2.6, n=4)[t(6)=0.416, p=0.692$ $\left.\eta^{2}=0.0281\right]$. e Representative images of neuromuscular junction (NMJ) immunofluorescence in whole mount (unsectioned) triangularis sterni muscles in 3-week and end-stage GA-Nes mice and Nes littermate controls, and microtome sectioned quadriceps muscles in end-stage GA-Nes mouse and Nes littermate control. Examples of denervated NMJs with loss of $\beta$-tubulin III (green) immunostaining within $\alpha$-bungarotoxin (red) labeled endplates are indicated with arrows. Scale bar $=50 \mu \mathrm{m}$. f Quantification of \% denervated neuromuscular junctions (NMJs) in the triangularis sterni shows that NMJs are unchanged at 3 weeks, but significantly denervated by end-stage in GA-Nes vs Nes littermate controls [Two-way ANOVA interaction (age $\times$ genotype) $F(1,11)=110, p<0.001 \eta^{2}=0.91 ; 3$ wk Nes $(0.02 \pm 0.004, n=3)$ vs GA-Nes $(0.02 \pm 0.006, n=4) \mathrm{ns} p>0.99$; endstage Nes $(0 \pm 0, n=4)$ vs GA-Nes $(79.3 \pm 13.9, n=4) * * * p<0.001]$. g Representative images of NMJs in the triangularis sterni muscle of single PR-Nes affected and asymptomatic mice, as well as littermate Nes controls. No denervation was observed in these samples

S5a). In the cortex of control mice, TDP-43 staining with an $\mathrm{N}$-terminal antibody showed the typical nuclear network-like pattern (Fig. 4a). TDP-43 expression seemed more diffuse in GA-Nes mice and to a lesser extent also in PR-Nes mice, but we did not observe nuclear clearance or cytoplasmic TDP-43 aggregates in GA-Nes and PR-Nes lines and did not observe additional cleavage products in the RIPA-insoluble fraction of GA-Nes mice by Western blot (Fig S5b). However, we noticed occasional nuclear TDP-43 aggregates ( $\sim 1 \%$ of neurons) specifically in the lateral frontal cortex and hippocampal CA regions of GA-Nes mice (Fig. 4a-c). In contrast, even in neurons with numerous nuclear poly-PR aggregates, no nuclear or cytoplasmic inclusions of TDP-43 were observed in asymptomatic or affected PR-Nes mice (Fig. 4a). We detected these nuclear TDP-43 inclusions in GA-Nes mice with several $\mathrm{N}$ - and C-terminal antibodies, and comparison of C-terminal antibodies specific for non-phospho- or phospho-TDP-43 showed that the nuclear aggregates are not phosphorylated at serine $403 / 404$ or serine 409/410 (Fig. S5a).

To further characterize the TDP-43 nuclear pathology in GA-Nes mice, we investigated co-aggregation of other RNA-binding proteins implicated in disease, as well as proteins involved in general RNA trafficking and splicing functions. Unexpectedly, immunostaining revealed strong colocalization of nuclear TDP-43 aggregates with hnRNP H, which has been linked to RNA toxicity in $C 9$ orf 72 cases, and weak colocalization with hnRNP A1 previously implicated in ALS/FTD [30, 33] (Fig. 4d). Absence of colocalization with the RNA-binding proteins FUS, SFPQ or ALYREF demonstrates the specificity of the interaction (Fig. 4d). Thus, only poly-GA promotes sparse nuclear aggregation of TDP-43 and other hnRNPs implicated in C9orf 72 disease in a region-specific manner.

\section{Poly-GA downregulates a network of synaptic functional genes and triggers pro-inflammatory responses}

To allow unbiased investigation of disease-related CNS pathomechanisms in our mouse models, we performed RNA sequencing of bulk tissue. For GA-Nes mice, we compared brainstem, cortex, hippocampus and spinal cord at end-stage and at 3 weeks (for the brain regions). For PR-Nes, we analyzed the cortex and hippocampus of 4-week affected and 68 -week-old asymptomatic mice. The full expression data are listed in Table S1.

Principal component analysis showed strong separation of the genotypes in different brain regions in GA-Nes endstage and affected PR-Nes mice, but not in GA-Nes mice at 3 weeks of age, which further excludes developmental effects due to poly-GA expression (Fig. S6a). Correlation analysis revealed overall similar gene expression changes in the different brain regions of GA-Nes mice particularly at the end-stage time point (Fig. S6b). We analyzed differential gene expression for all regions and time points with DESeq2. In total 2351 genes were significantly up- or downregulated in GA-Nes mice, compared with only 123 genes in PR-Nes mice and 53 in both using our cut-off criteria (adjusted $p<0.05$, see Table S2). The genes showing the strongest differential expression in GA-Nes and PR-Nes are shown in Fig. 5a. We detected the strongest changes in the GA-Nes hippocampus, in line with the early pronounced microglial activation and neuron loss visible in this area (compare Fig. 2f). The strongest change in PR-Nes mice is the reduced expression of three 5S rRNA transcripts in the cortex of both affected and asymptomatic mice, which corroborates the known effects of poly-PR in inhibiting protein translation [21]. Among the top differentially expressed genes, only $\mathrm{Ccl} 2$ and $\mathrm{Cxcl} 10$ were significantly different in both GA-Nes and PR-Nes mice. Gene expression changes 

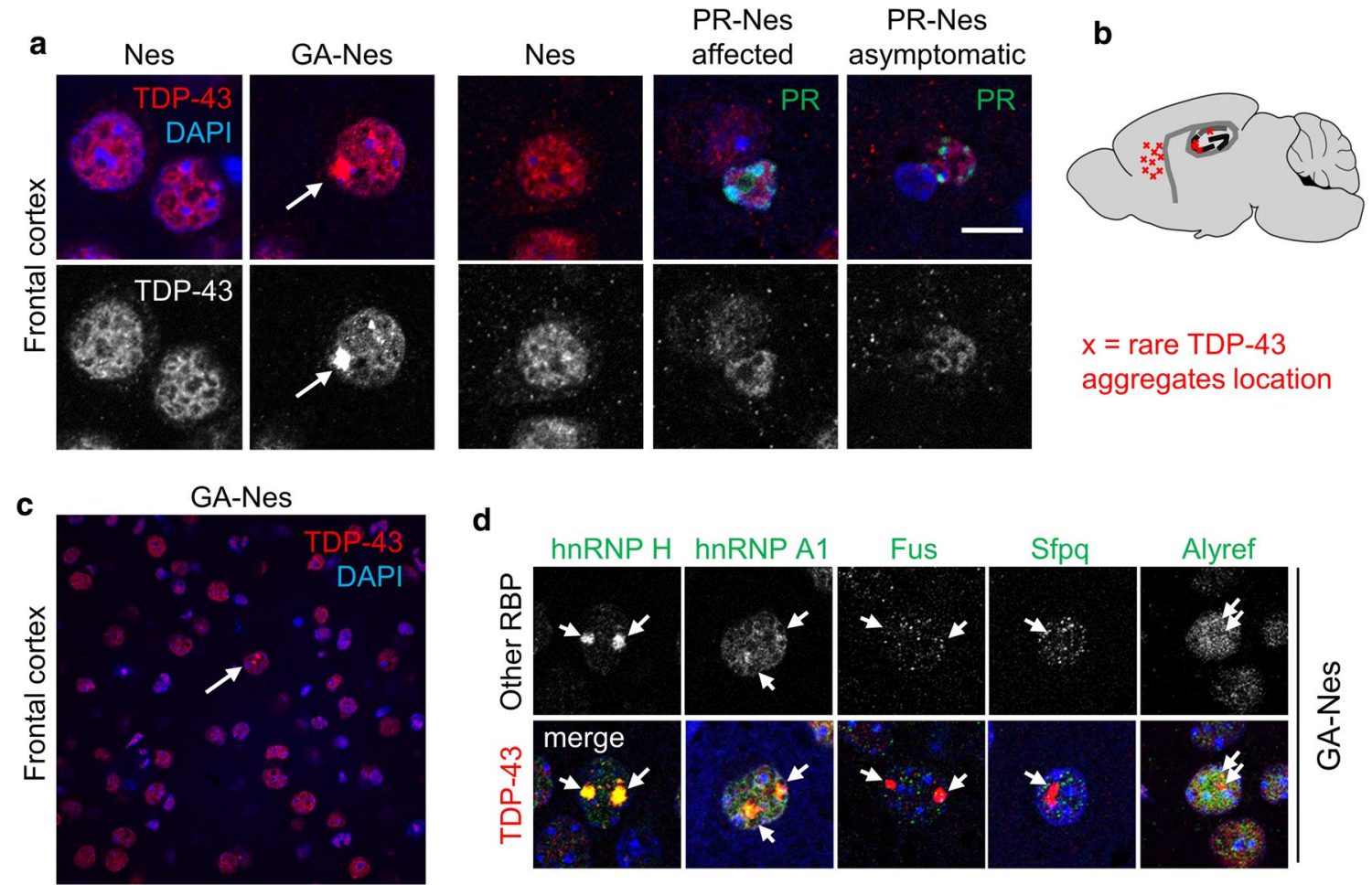

d
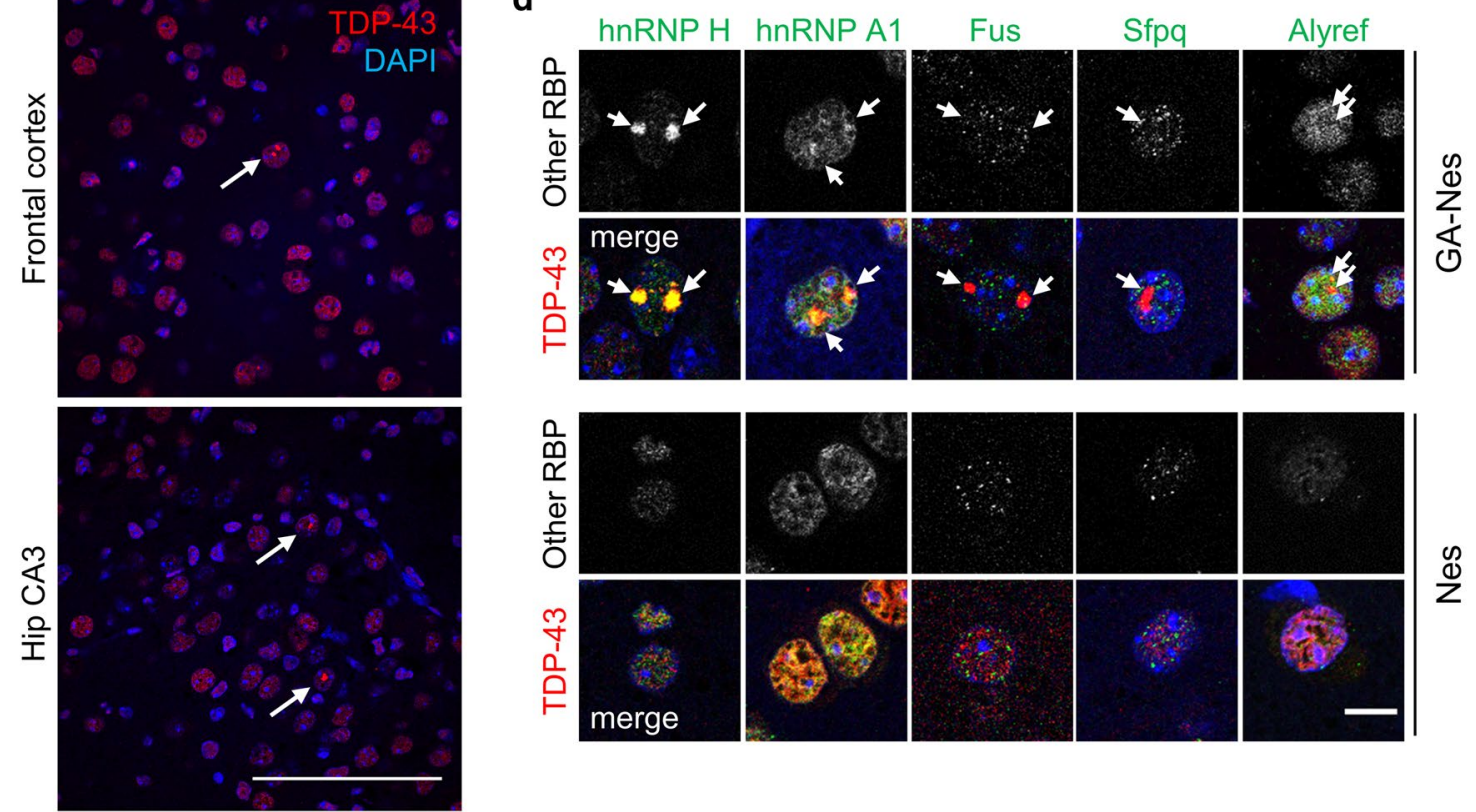

Fig. 4 TDP-43 and other disease-linked RNA-binding proteins form nuclear inclusions in GA-Nes mice. a Neuronal nuclear inclusions of TDP-43 (arrow) are detected by immunofluorescence in hippocampus and frontotemporal cortex in GA-Nes end-stage mice, but not Nes littermate controls. No TDP-43 inclusions are detected in affected or asymptomatic PR-Nes mice, or a 68-week Nes control, even in cells with abundant poly-PR (green) inclusions. Scale bar $=10 \mu \mathrm{m}$. b Schematic drawing of the distribution of the rare nuclear TDP-43 aggregates in GA-Nes mice. Aggregates were found in the lateral

frontal cortex (at least $100 \mu \mathrm{m}$ from the midline) and hippocampal CA regions marked with red $x$. c Regional overview of TDP-43 nuclear aggregates (arrows) in the frontal cortex and hippocampus CA3 region of end-stage GA-Nes mice, scale bar $=100 \mu \mathrm{m}$. d Nuclear TDP-43 aggregates (arrows) in end-stage GA-Nes mice colocalize with hnRNP H, and weakly with hnRNP A1, but not with Fus, Sfpq or Alyref. No nuclear aggregates of any marker are seen in agematched Nes mice. Scale bar $=10 \mu \mathrm{m}$

in asymptomatic PR-Nes mice are minimal and revealed no obvious mechanistic basis for the transgene repression in asymptomatic compared to affected mice (Table S1).

Among down-regulated transcripts, gene ontology (GO) analysis identified enrichment of genes encoding synaptic proteins (e.g., neurotransmitter receptor subunits and proteins involved in vesicle release) as well as immediate early genes associated with learning and memory in GA-Nes cortex and hippocampus at end-stage, but not in PR-Nes tissues (Fig. 5b, c). Comparison of significant hits (bold), related subunits of GABA, NMDA and dopamine receptors and other key synaptic genes suggests altered synaptic function in GA-Nes mice may result in reduced activation of immediate early genes such as Arc, which is critical for learning and memory [42] (Fig. 5c). Analysis of protein levels in neocortex by western blot showed that the immediate early gene Arc is indeed significantly reduced in the end-stage cortex of GA-Nes mice, but levels of key neuronal proteins $\beta$-tubulinIII and PSD-95 were not reduced (Fig. 5d, e). These findings are consistent with the absence of neuron loss in this region by the time of euthanasia (see Fig. 2i). Myelination-associated genes (Klk6, Mal, Pllp, Plp1, Fa2h, Hes5, Aspa) were 
also downregulated in GA-Nes and PR-Nes mice, likely due to DPR expression in a subset of oligodendrocytes (see Fig. S1f), which has so far not been reported in C9orf72 patients.

Overall, up-regulated transcripts in GA-Nes mice were strongly enriched for immune responses, including cytokineand chemokine-mediated signaling and interferon-inducible genes such as Ifi205/207 and chemokines in all regions in end-stage GA-Nes but not (or much less) PR-Nes mice (Fig. 5a, Table S3). Thus, poly-GA expression induces pronounced neuroinflammation and may also impair synaptic function.

\section{Poly-GA triggers microglial interferon responses enriched in C9orf72 ALS}

To examine the relevance of these findings to human disease, we correlated gene expression changes in GA-Nes mice with large public transcriptome data of spinal cord, motor cortex and cerebellum from ALS patients (TargetALS). All regions showed clear expression changes in ALS cases (see full expression data in Table S4). Surprisingly, gene expression changes in all GA-Nes regions correlated better with spinal cord than motor cortex from both $C 9$ orf 72 and non- $C 9$ orf 72 ALS patients, and inversely correlated with cerebellum and frontal cortex (Fig. S6c). In contrast, PR-Nes mice showed only weak correlations with human ALS tissue. Comparing gene expression in C9orf72-positive and C9orf72-negative cases (C9vsALS), confirmed the upregulation of homeobox genes in the cerebellum (e.g., HOXA5 [15]) and showed altered expression of genes related to the extracellular matrix in the spinal cord of C9orf72 cases (Table S5 and Fig. 6a). GO analysis of genes concordantly regulated in GA-Nes mice and the C9vsALS patient data also showed enrichment of interferon signaling (e.g., IRF7 and IRF9) and defense response genes (e.g., GRN), while no GO terms were concordantly enriched in both PR-Nes and C9vsALS using our criteria (Fig. 6a). The network of these immunerelated genes concordantly upregulated in GA-Nes mice and C9vsALS brains is shown in Fig. 6 .

To further verify whether the pro-inflammatory responses were driven by microglia, we performed RNA sequencing on isolated microglia from end-stage GA-Nes brain. Transgenespecific sequence tags (GA175) further confirm that polyGA is not expressed in microglia (Fig. S6d). The top differentially expressed genes in GA-Nes microglia include many interferon-stimulated genes (e.g., MX1, Isg 15, Oasl1/2 and various Ifi genes) and complement factors ( $\mathrm{C} 3$ and $\mathrm{C} 4 \mathrm{~b}$ ) (Fig. S6d). Therefore, we further compared our data to different microglial subtypes recently identified by single-cell transcriptome studies of microglia under physiological and pathological conditions $[19,40]$. The strongest positive correlations were with cluster 6 from a p 25 neurodegeneration model [40] and in a lysolecithin demyelination model (injury 2) [19], two microglia subpopulations with strong induction of interferon-response genes (Fig. 6c). Comparing this with the bulk tissue sequencing, these signatures also correlate moderately in GA-Nes end-stage tissues, but weakly or not at all in PR-Nes (Fig. 6c). Comparing only the signature genes of several published microglia populations shows the greatest overlap with interferon-response microglia (IRM) in a mouse model of Alzheimer's disease [56] (Fig. S6e). Together, these data indicate that poly-GA expression induces a proinflammatory microglial response dominated by interferon signaling.

We confirmed the interferon response in end-stage GANes mice by immunoblotting for the STAT proteins that act as intermediate effectors of interferon signaling. The upregulation of total STAT1 and STAT3 suggests strong microglial proliferation, and the upregulation of phosphorylated STAT3 relative to total in GA-Nes cortex (Fig. 6d, e) is consistent with an interferon response. Stat 3 gene expression was also significantly elevated at end-stage in hippocampus and cortex (Table S2). Measuring IFN $\gamma$ directly by ELISA showed that IFN $\gamma$ was significantly elevated in end-stage GA-Nes but not affected PR-Nes mice (Fig. 6f, g). Taken together, our data indicate that poly-GA inclusions trigger interferon responses in microglia that are also enriched in human C9orf72 ALS.

\section{Discussion}

Using novel mouse models for conditional high-level expression of key DPRs, we show that poly-GA triggers rapidly progressive neuroinflammation, muscle denervation, and selective neuronal loss in all mice. A congenic poly-PR line with the same repeat length and similar mRNA levels produced far lower DPR aggregation resulting in ataxia and seizures requiring early euthanasia in a subset of animals. However, $60 \%$ of animals were able to suppress poly-PR mRNA expression to non-toxic levels, which importantly were still higher than the frequency of poly-PR reported in human tissues $[38,58]$. In addition, we found no clear ALS/ FTD-related pathology in either affected or asymptomatic PR-Nes mice. Thus, our comparative study highlights the role of poly-GA in C9orf72 ALS/FTD by triggering neuron loss and neuroinflammation, and our GA-Nes mouse model will allow rapid testing of poly-GA-directed therapy in the future.

\section{Differential aggregation and toxicity of poly-GA and poly-PR in vivo}

Poly-PR is highly toxic in mammalian cells, yeast and drosophila by interfering with stress granule formation, nucleocytoplasmic transport, heterochromatin 
a

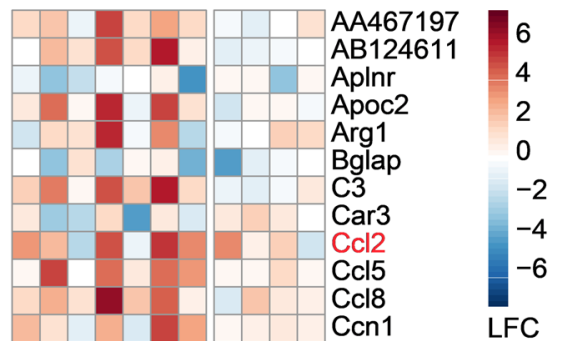

LCn1 LC

Cd300lf

Cd72

Cd74

Cep55

Clec7a

Csf2rb

Cst7

Cxcl10

Cyp4f18

Endou

Fcgr4

FInc

GA175

Gm44805

Gm49339

H2-Q6

Hsp25-ps1

Hspb1

Ifi205

Ifi207

Itgad

Lcn2

Mboat1

Milr1

Mki67

Mmp12

Msr1

n-R5s 118

n-R5s193

n-R5s194

Nuf2

Osmr

Pbk

Pclaf

Pimreg

Pirb

Plin4

PR175

$\mathrm{Prl}$

Saa3

Serpina3i

Serpina3m

Slc43a3

Socs 3

Sprr1a

Steap4

Tgm1

Timp1

Top2a

Zbe2c

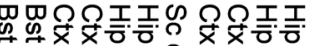

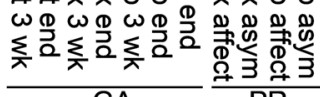

b

neutrophil chemotaxis mitotic cell cycle phase transtion down up

transition myelination : :

negative regulation of immune system process T cell activation. cytokine-mediated signaling pathway
myeloid leukocyte activation

positive regulation of defense response

regulation of ERK1 and ERK2 cascade

positive regulation of cytokine production antigen processing and presentation. response to interferon-gamma monocyte chemotaxis response to chemokine chemokine-mediated signaling pathway. regulation of immune effector process negative regulation of protein phosphorylation

ossification -

carboxylic acid biosynthetic process synaptic transmission. GABAergic. neurotransmitter transport multicellular organismal response to stress. regulation of synaptic vesicle exocytosis. regulation of membrane potential muscle system process monovalent inorganic cation transport. memory

C
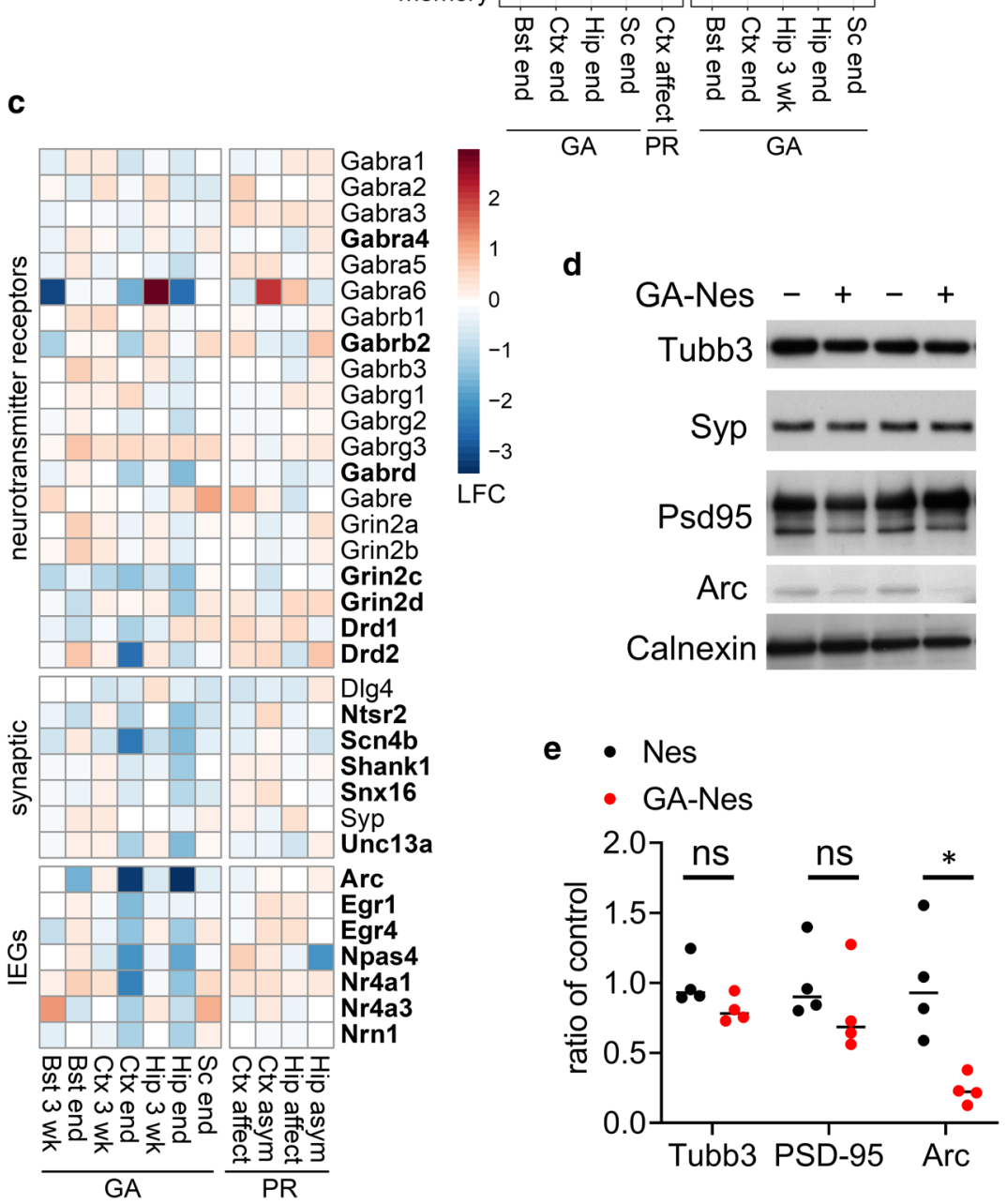

d

GA-Nes - + - +
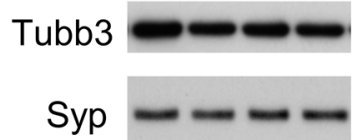

Psd95 20

Arc

Calnexin

e Nes

- GA-Nes

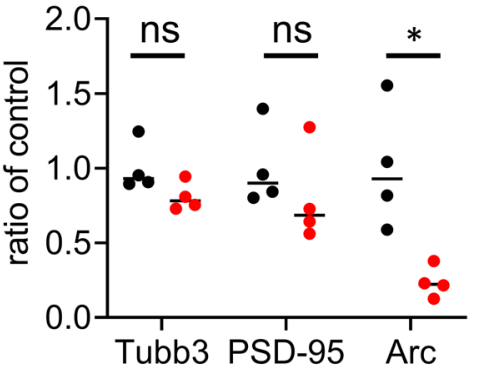

organization, nucleolar dynamics and protein translation by affecting the liquid-liquid phase separation of other low-complexity proteins $[2,3,21,24,31,32,44$, 68]. While poly-PR localized to heterochromatin and the nucleolus in both affected and asymptomatic PR-Nes mice as in other models, we observed no neuron loss in PR-Nes animals. In addition, asymptomatic PR-Nes mice seem to escape lethality by suppressing the expression of the synthetic transgene mRNA. Thus, nuclear poly-PR toxicity is dose dependent, consistent with another recent report [20]. 
४Fig. 5 Poly-GA reduces many genes important for synaptic function and triggers inflammation. a Significantly regulated genes in GA-Nes and PR-Nes with absolute $\log _{2}$-fold expression changes larger than 4.5 in at least one condition. Significant differences are in PR-Nes mice are noted in green, and those overlapping between GA-Nes and PR-Nes are noted in red. The remaining genes are only differentially expressed in GA-Nes, predominantly at end-stage. For the full list of differentially expressed genes, see Tables $\mathrm{S} 1$ and $\mathrm{S} 2 \mathrm{a} / \mathrm{b}$. b Gene ontology analysis of differentially expressed genes (compare Tables S2 and S3) for the indicated regions and time points. The top 5 nonoverlapping terms with more than 5 genes are shown for each group (groups that do not meet these criteria are not shown). The largest cluster among downregulated genes is related to synaptic function in GA-Nes, and among upregulated genes is related to immune response in GA-Nes. Genes per group from left to right: 193, 226, 264, 50, 58, $372,585,42,991,90$. The dot size and color represent the fraction of the differentially expressed genes in each category and adjusted $p$ values, respectively. $\mathbf{c}$ Heatmap of genes in the synaptic terms (including membrane potential and memory) identified in $\mathbf{b}$ are most consistently reduced in GA-Nes Ctx and Hip at end-stage, but not at 3 weeks or in PR-Nes. Bold genes are differentially expressed in at least one condition (adjusted $p<0.05$ ). d Representative western blots from the neocortex show reduced Arc levels, but no change in selected neuronal proteins in end-stage GA-Nes (+) vs. Nes controls (-). Calnexin is used as a loading control. e Protein quantification of neuronal markers in d, $\beta$-tubulin III (Tubb3) and PSD-95 (Dlg4) shows no significant change in protein levels in end-stage GA-Nes mice [Tubb3: Mann-Whitney $t$ test $U=2$, ns $p=0.114$; PDS-95 $t$ test $t(6)=0.939$, ns $p=0.384]$, while functional marker Arc is significantly reduced [Welch's $t$ test $t(3.39)=3.58,{ }^{*} p=0.03$ ]. $n=4$ /group

In addition, post-transcriptional suppression mechanisms likely exist in vivo, because most neurons outside the hippocampus in affected PR-Nes mice have no detectable poly-PR aggregation. Indeed, we find reduced expression of 5S rRNA in the cortex but not hippocampus in PR-Nes mice that may contribute to reduced poly-PR protein levels in the cortex, and is consistent with poly-GR/PR-mediated inhibition of translation [21, 25, 35, 66].

In contrast, poly-GA shows modest toxicity in rodent primary neurons by gradually sequestering the proteasome and other proteins $[18,41]$. We confirmed sequestration of the proteasome in our GA-Nes mice and occasional nuclear aggregation of TDP-43 and other low-complexity RNA-binding proteins suggests poly-GA impairs proteostasis in vivo, as we showed recently in vitro $[18,29]$. Surprisingly, using identical expression systems, poly-GA toxicity and aggregation was more severe in the mouse central nervous system and resulted in a more ALS-like phenotype than poly-PR expression. Proteasomal impairment, indicated by partial sequestration of Psmc4 in GANes, may contribute to the strong accumulation of polyGA compared to poly-PR despite similar mRNA levels in affected PR-Nes mice, consistent with data from cellular models [41]. Overall, direct comparison of our mouse lines indicates a combination of post-transcriptional mechanisms could explain the predominant aggregation of poly-GA compared to poly-PR seen in $C 9$ orf 72 patients without invoking RAN translation-mediated effects.

\section{Widespread poly-GA but not poly-PR expression causes disease-relevant phenotypes}

In our models, poly-GA led to selective neuron loss in the hippocampus and spinal cord as well as muscle denervation resembling ALS. In contrast, poly-PR expressed at the same mRNA level led to ataxia and seizures without obvious neuron loss or inflammation in the brain or spinal cord. While these findings confirm toxicity of high nuclear polyPR levels, they do not replicate ALS phenotypes well. However, since national animal welfare regulations required us to euthanize mice after a single stage II seizure (see "Materials and methods"), we cannot exclude that repeated seizures would lead to overt neuron loss in these mice. More ALS-like toxicity of poly-PR has been observed in AAVbased expression models or with homozygous transgenic expression that drives higher poly-PR levels compared to our single-copy transgenic line, leading to weight loss and early lethality in a subset or all mice, respectively [20,68]. Both of these poly-PR mouse models also showed ataxialike phenotypes due to Purkinje cell loss, which was not detected in our PR-Nes model (data not shown), and one line showed loss of lower motor neurons and inflammation within the spinal cord [20]. In contrast, in our PR-Nes line, poly-PR inclusions are enriched in the hippocampus, a common focal point of epilepsy and the area where polyPR aggregates have been reported to be most abundant in human $C 9$ orf 72 FTD [38, 58]. However, while seizures and epilepsy have been reported in $C 9$ orf 72 patients, they are not a common feature [5]. The different phenotypes of low and high-expressing PR-Nes mice further support dosedependent toxicity of poly-PR. However, even asymptomatic PR-Nes mice have more abundant poly-PR inclusions than C9orf72 patients [38, 58]. While the scarcity of poly-PR inclusions in patients has been widely attributed to its high toxicity in vitro $[62,68]$, the modest transcriptional changes in PR-Nes mice despite Nestin-Cre-driven expression of the poly-PR transgene throughout the CNS under the control of the strong CAG promoter do not support dominant effects of barely detectable poly-PR aggregates in patients. Nevertheless, we cannot rule out that the predominantly cytoplasmic localization of poly-PR in patients $[16,58]$ and the longer repeat length could still cause more dramatic effects in patients.

Overall, our poly-GA mice model several key aspects of C9orf72 ALS well, including selective loss of motor neurons in the spinal cord, muscle denervation, and an interferon-response microglial signature concordant with C9orf72 ALS. In addition, the sparse nuclear TDP-43 inclusions only in the forebrain are reminiscent of $C 9$ orf 72 

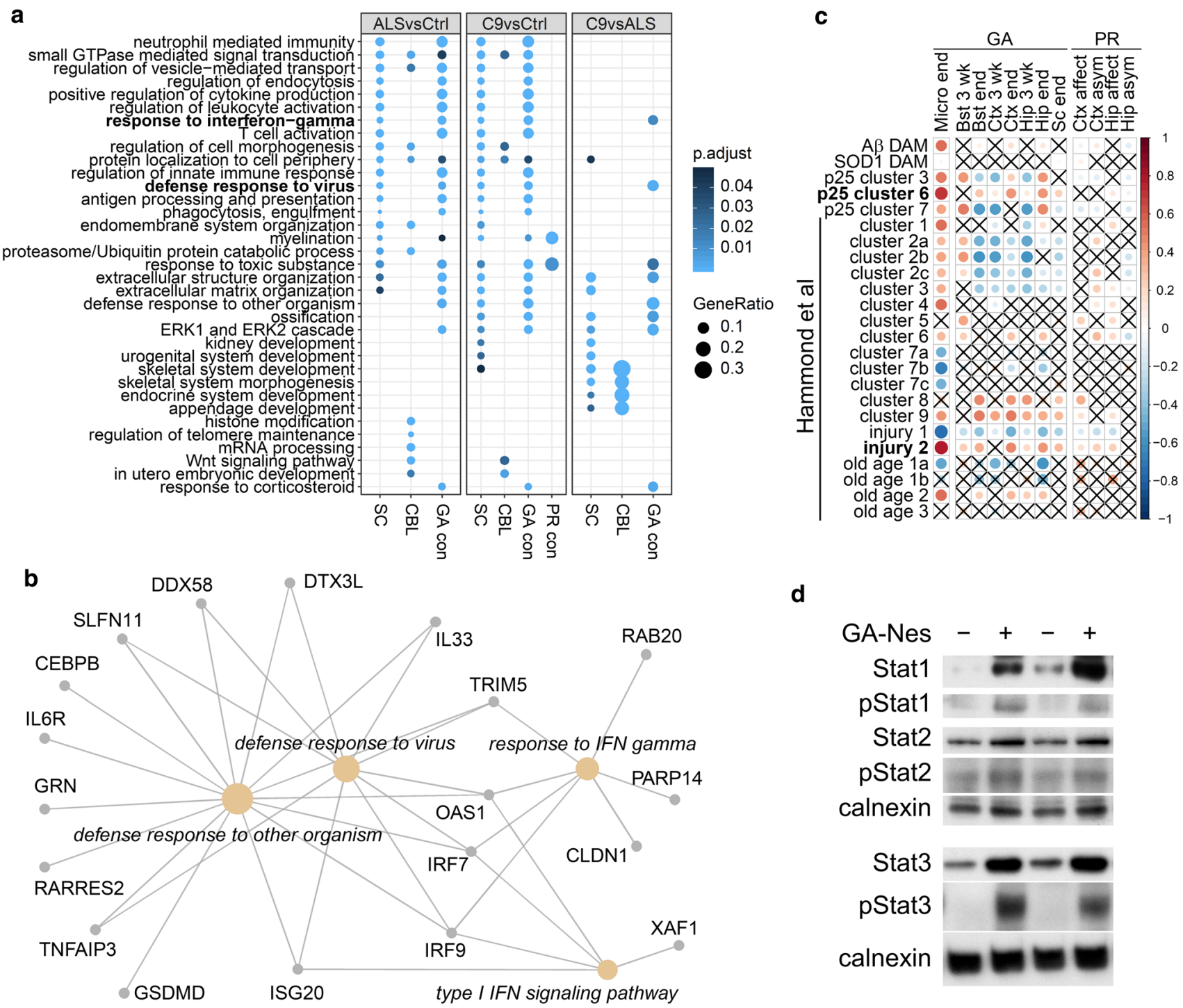

\section{d}
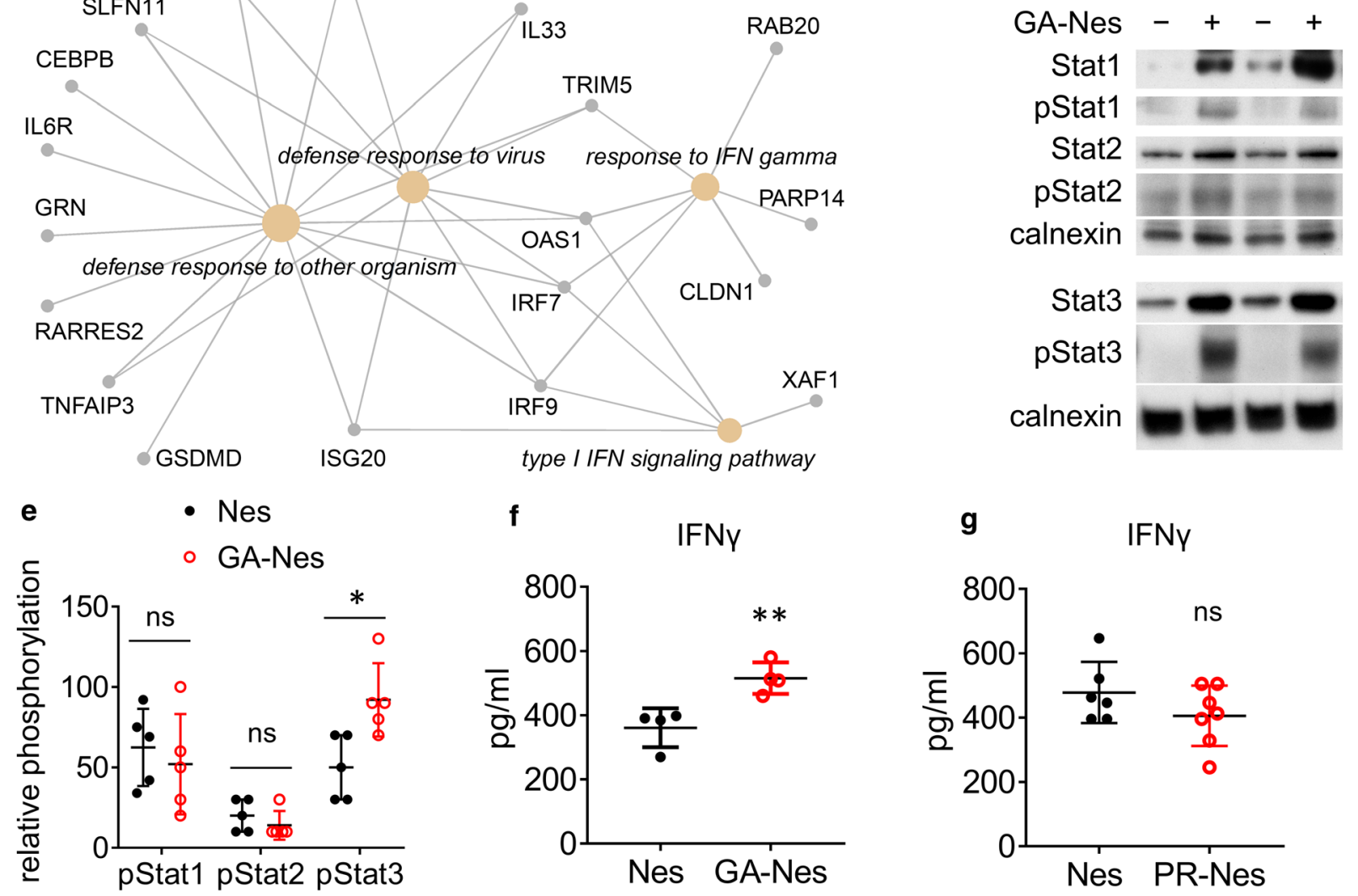

FTD. Together these data provide in vivo evidence for selective vulnerability towards poly-GA that may explain the disconnect between $C 9$ orf 72 -specific pathology and neurodegeneration in patients. However, it is necessary to understand the three limitations of this model to maximize its usefulness. First, our mice show modest poly-GA expression in some cells in kidney, pancreas and GI tract, which has not been reported in C9orf72 patients [1]. While histopathological analysis nevertheless revealed overall normal organ morphology and poly-GA expression in the neurons of the myenteric plexus of GA-Nes mice does not seem to impair peristalsis (based on observation 
४Fig. 6 Poly-GA, but not poly-PR, triggers microglial pro-inflammatory responses characteristic of disease-associated microglia, aging, and interferon that are similar to C9orf72 ALS patients. a Gene ontology analysis of genes differentially expressed between ALS cases without ("ALS") or with C9orf72 ("C9") mutation compared to controls ("Ctrl"). Terms with $>5$ genes that are concordantly regulated in GA-Nes (GA con) or affected PR-Nes (PR con) mice are also analyzed. Concordantly regulated genes were defined by comparing averaged significant up/down-regulation from all analyzed regions in GA-Nes or affected PR-Nes mice and ALS patients. Number of genes in each group from left to right: 5810, 4742, 660, 5351, 1279, 689, 45, 999, 33, 111. SC spinal cord, CBL cerebellum. Full data is available in Table S4 and S5. b Network of the immunerelated genes concordantly regulated in GA-Nes mice and C9orf72positive vs C9orf72-negative ALS patients. c Correlation analysis of gene expression in isolated microglia from GA-Nes mice or whole tissue from different brain regions in GA-Nes- and PR-Nes-affected (affect) and asymptomatic (asym) mice with differentially expressed genes in microglial subpopulations identified by single-cell RNAseq in $\mathrm{A} \beta$ and SOD1 mouse models [26], p25 neurodegeneration mice [40] and wildtype mice during development (cluster 1-9), injury and aging [19]. Pearson's correlation coefficient indicated by color and circle size. Non-significant correlations are crossed out. Size of the published genes sets from top to bottom: 1482, 4589, 730, 955, 271, $278,332,483,295,678,452,118,158,40,83,52,66,183,192,188$, $22,12,130,36$. Strongest positive correlation with GA-Nes microglia is found in interferon-response clusters injury 2 and p 25 cluster 6. d Immunoblots from whole neocortex of total and phosphorylated Stat effector proteins of interferon signaling in end-stage GA-Nes $(+)$ and age-matched Nes controls (-). Calnexin is used as a loading control. e Relative phosphorylation levels normalized to total protein levels increase in end-stage GA-Nes only for Stat3 compared to age-matched Nes controls [Two-way ANOVA (effector $\times$ genotype) $F(2,24)=3.88, p=0.035 \eta^{2}=0.323$. Sidak post hoc pStat 1 Nes $(62.4 \pm 24.0)$ vs GA-Nes $(52 \pm 31.1)$ ns $p=0.858$, pStat2 Nes $(20 \pm 10)$ vs GA-Nes $(14 \pm 8.9)$ ns $p=0.998$, pStat3 Nes $(38.6 \pm 17.3)$ vs GA-Nes $(82.6 \pm 24.9) * p=0.017], n=5 /$ group. f ELISA for IFN $\gamma$ in whole neocortex homogenate is significantly increased in endstage GA-Nes mice. [Mann-Whitney Nes $(361 \pm 60.7)$ vs GA-Nes $(515 \pm 49.3) * * p=0.029,97 \%$ CI $62.3-310] n=4 /$ group. g IFN $\gamma$ in whole neocortex homogenate is not significantly changed in affected PR-Nes mice compared to Nes littermate controls. $[t(10)=1.661$, Nes $(478 \pm 94.9)$ vs PR-Nes affected $\left.(389 \pm 91), p=0.128 \eta^{2}=0.216\right]$ $n=6 /$ group. Power to detect $\eta^{2}>0.5=83.7 \%$

of the intact GI tract), we cannot rule out more subtle functional deficits. Investigating whether DPR inclusions occur in the enteric nervous system of C 9 orf 72 patients could be worthwhile because cachexia is a common but poorly understood feature of sporadic and C9orf72 ALS that often precedes the diagnosis by many years [52]. Second, although the distribution of DPR inclusions is predominantly neuronal a subset of oligodendrocytes and astrocytes do express transgene RNA. Third, GFP-only control littermates would have been ideal to rule out any effects of GFP alone, but this was not possible with our construct design. However, we are not aware that any of the large number of published mouse lines expressing high levels of GFP in the brain have shown phenotypes similar to our GA-Nes mice. Moreover, the largely nonoverlapping phenotypes of our GA-Nes and PR-Nes mice argue against non-specific off-target effects due to the widespread expression of DPR proteins or GFP using the Nestin-Cre driver.

An important aspect of our novel poly-GA mouse model is that all mice reached the predefined endpoints according to national animal welfare regulations before 7 weeks, while abundant data from the neuromuscular junctions, motor neurons, and transcript analysis indicate no developmental defects. This consistent and rapid progression with motoneuron loss, denervation and C9orf72-enriched transcriptional changes are unique features of this $C 9$ orf 72 model and offer an advantage for analyzing poly-GA-targeted therapy in vivo. As in other ALS models and patients, denervation is more pronounced than motor neuron loss arguing for the contribution of dying-back mechanisms due to synaptic toxicity [10]. In addition, poly-GA expression in muscle tissue could contribute to denervation and weakness in GA-Nes mice, and recent studies have uncovered that poly-GA is expressed in the skeletal muscle of C9orf72 patients [9]. Crossing our conditional line with other Cre drivers will allow us to dissect the role of peripheral poly-GA expression in the future.

\section{TDP-43 and other disease-linked RNA-binding proteins form nuclear inclusions in GA-Nes mice}

In addition to DPR inclusions, TDP-43 inclusions are characteristic of sporadic and C9orf72-related and other forms of ALS/FTD and correlate far better with neurodegeneration [37]. However, it is still debated how TDP-43 pathology is triggered by the C9orf72 mutation. The lack of spatial correlation of DPR expression, RNA foci and C9orf72 expression with TDP-43 aggregates in patients suggests that selective vulnerability is critical. This is supported by the striking finding that nuclear TDP-43 aggregates selectively in a subset of neurons in the hippocampus and frontal cortex of GA-Nes mice, despite abundant poly-GA aggregation throughout the brain. Study of the pathomechanism in mice is complicated by differences between mouse and human molecular biology, in particular the processing by caspase 4 that generates the aggregation-prone C-terminal fragments found in cytoplasmic inclusions [64]. Rare cytoplasmic inclusions of TDP-43 have been reported in some $\left(\mathrm{G}_{4} \mathrm{C}_{2}\right)_{n}$ models and viral poly-GA expressing mouse models, but most inclusions in these mice are nuclear [6, 23, 34, 66]. In our congenic mouse models, only poly-GA induced regional intranuclear aggregates of TDP-43 that were not phosphorylated at the disease-specific sites S409/410 or S403/404. We did not observe disease-like proteolytic processing of TDP43 or cytoplasmic TDP-43 inclusions. Nevertheless, the nuclear TDP-43 aggregates partially colocalize with other RNA-binding proteins hnRNP $\mathrm{H}$ identified as an interactor of $\left(\mathrm{G}_{4} \mathrm{C}_{2}\right)_{n}$ RNA, and hnRNP A1 also implicated in ALS/ 
FTD $[8,30,33]$. We now show that poly-GA alone is sufficient to induce aggregation of hnRNP $\mathrm{H}$, in the absence of $\left(\mathrm{G}_{4} \mathrm{C}_{2}\right)_{n}$ RNA. Overall, although these nuclear inclusions are rare at the young age of our mice, our data suggest that poly-GA impairs proteostasis of disease-linked RNA-binding proteins, perhaps mediated by proteasome sequestration $[18,29]$. Together with other insults such as defective RNA transport and caspase-mediated cleavage, this may ultimately lead to their cytoplasmic aggregation in patients.

\section{Poly-GA triggers pro-inflammatory interferon responses similar to human ALS spinal cord}

GA-Nes mice show strong activation of microglia throughout the CNS, but particularly in the hippocampus where neurons are lost at an early age. We used RNAseq to elucidate the inflammatory response and conducted a time-course study in multiple CNS regions in GA-Nes and PR-Nes mice. The gene expression profiles are dominated by chemokines and other inflammatory changes in GA-Nes mice with the strongest effects in the degenerating hippocampus. In addition, synaptic genes and several immediate early genes such as Arc, which is crucial for memory formation [42], are downregulated. Together with our observation that cortical neurons are not lost by this age in GA-Nes mice, these data suggest that poly-GA impairs synaptic function, which may contribute to $C 9$ orf 72 pathogenesis prior to overt neuron loss [54].

Comparing differential gene expression in GA-Nes mice and human ALS cases shows a strong overlap with an inflammatory signature common to both $\mathrm{C} 9$ orf 72 and sporadic ALS. Comparing gene expression in GA-Nes mice and $C 9$ orf 72 -specific changes in human ALS spinal cord highlights the role of enhanced interferon signaling. Interestingly, IFN $\alpha$ levels are higher in the CSF of C9orf72 ALS compared to sporadic ALS [51]. Transcriptome analysis in cortex samples from ALS cases identified a subgroup of patients with high microglia/interferon response, without enrichment in C9orf72 cases, but the spinal cord was not analyzed in that study [60]. Comparing gene expression of isolated GA-Nes microglia to recently published microglia expression signatures from single cell RNAseq experiments $[19,26,40,56]$ highlights the role of pro-inflammatory microglia subpopulations characterized by a strong interferon response. Indeed, many of the differentially expressed genes are known to be interferon-inducible and we show that STAT signaling is enhanced in GA-Nes mice. In addition, we confirmed that IFN $\gamma$ was increased in GA-Nes but not PR-Nes mice. Moreover, CHIT1, a sensitive CSF biomarker for ALS [50, 59] is also strongly induced by interferon [39] suggesting a sterile interferon response is highly relevant in human ALS. A causal role of interferon in neurodegeneration is supported by inherited interferonopathies such as Aicardi-Goutieres syndrome, associated with cerebral atrophy and psychomotor retardation in children [17]. We show for the first time that poly-GA is sufficient to induce strong interferon responses in vivo, which is seen in the spinal cord of sporadic ALS and enriched in C9orf72 patients. Future experiments to investigate whether inhibiting this pro-inflammatory response will extend the lifespan of GANes animals would help determine whether this may be a therapeutic approach for $C 9$ orf 72 ALS.

\section{Conclusion}

Taken together, our GA-Nes mice reproduce key features of $C 9$ orf72 ALS/FTD far better than the congenic PR-Nes mice. In addition, we identify a C9orf72-enriched inflammatory signature triggered by poly-GA but not poly-PR in mice that supports a dominant role of poly-GA in C9orf72 ALS/ FTD pathogenesis. In contrast, the low abundance of poly$\mathrm{PR}$ aggregates in patients and post-transcriptional reduction of poly-PR in most mouse tissues (perhaps through downregulation of 5S rRNA) suggests that neurons in humans may also be capable of suppressing poly-PR aggregation and emphasizes the link to ribosome-mediated toxicity. Since poly-GA triggers region-specific neuron loss and down-regulation of synaptic genes essential for memory and induces an inflammatory milieu similar to ALS spinal cord, we propose poly-GA and subsequent inflammatory responses are important targets for C9orf72 therapeutics. Despite some limitations, our novel model for C9orf72 ALS/FTD with widespread high-level poly-GA expression, selective vulnerability in spinal cord motor neurons, early onset and rapid progression will be useful for the initial preclinical testing of therapies to block poly-GA toxicity and associated proinflammatory interferon responses in microglia.

Acknowledgements Open Access funding provided by Projekt DEAL. We thank Zeynep Gunes, Marcel Matt, Sabine Odoy, Irina Pigur, Ali Rezaei and Michael Schmidt for technical support. We thank Kristin Rentzsch for cloning the plasmids for transgenic expression and Regina Kneuttinger for excellent technical assistance in the generation of poly-GA/PR transgenic mice. We thank Annette Feuchtinger at the Pathology and Tissue Analytics Core Facility of the Helmholtz Zentrum München for performing the organ pathology analysis. We thank Anja Capell, Andreas Hermann, MinAe Lee-Kirsch, Manuela Schneider and Sabina Tahirovic for critical comments to the manuscript. We thank Rüdiger Klein for providing Nestin-Cre mice. This work was supported by the Hans und Ilse Breuer Foundation and NOMIS foundation (D.E., M.N., K.D.L.), the Munich Cluster of Systems Neurology (SyNergy) (DFG EXC 2145 / ID 390857198 to D.E., T.A., T.M., M.B.S., W.W., Q.Z.), the European Community's Health Seventh Framework Programme under grant agreement 617198 [DPRMODELS] (D.E.) and 616791 [DIABLo] (T.M.). T.M.'s lab was further supported by the Deutsche Forschungsgemeinschaft via FOR 2879 (Mi694/9-1), CRC870 (A11) and TRR274 (B03, C02). M.H.d.A. is supported by the German Federal Ministry of Education and Research (Infrafrontier grant 01KX1012) and the German Center for Diabetes 
Research (DZD). We very much appreciate the gene expression data from human ALS cases and control provided by the Target ALS Human Postmortem Tissue Core, New York Genome Center for Genomics of Neurodegenerative Disease, Amyotrophic Lateral Sclerosis Association and TOW Foundation.

Author contributions $\mathrm{KDL}$ and QZ performed most mouse experiments with the assistance of MM, MC, MSB and TM. BW and WW generated the transgenic mice. AJ and WE performed RNAseq in transgenic mice. DE analyzed RNAseq data in mice and humans. BR and MHA performed hematology analysis. DF generated reagents. MN provided reagents and advice. TA counseled all pathology experiments. DE, TA, WW and WE supervised research. DE designed the study. DE and KDL wrote the manuscript. All authors discussed the data and the manuscript.

\section{Compliance with ethical standards}

Conflict of interest DE and TA hold a patent (US10066007B2) on "Dipeptide-repeat proteins as therapeutic target in neurodegenerative diseases with hexanucleotide repeat expansion". DE and QZ applied for a patent on active vaccination targeting DPRs in C9orf72 ALS/ FTD.

Open Access This article is licensed under a Creative Commons Attribution 4.0 International License, which permits use, sharing, adaptation, distribution and reproduction in any medium or format, as long as you give appropriate credit to the original author(s) and the source, provide a link to the Creative Commons licence, and indicate if changes were made. The images or other third party material in this article are included in the article's Creative Commons licence, unless indicated otherwise in a credit line to the material. If material is not included in the article's Creative Commons licence and your intended use is not permitted by statutory regulation or exceeds the permitted use, you will need to obtain permission directly from the copyright holder. To view a copy of this licence, visit http://creativecommons.org/licenses/by/4.0/.

\section{References}

1. Ash PE, Bieniek KF, Gendron TF, Caulfield T, Lin WL, Dejesus-Hernandez $\mathrm{M}$ et al (2013) Unconventional translation of C9ORF72 GGGGCC expansion generates insoluble polypeptides specific to c9FTD/ALS. Neuron 77:639-646. https://doi. org/10.1016/j.neuron.2013.02.004

2. Boeynaems S, Bogaert E, Kovacs D, Konijnenberg A, Timmerman E, Volkov A et al (2017) Phase separation of C9orf72 dipeptide repeats perturbs stress granule dynamics. Mol Cell 65(10441055):e1045. https://doi.org/10.1016/j.molcel.2017.02.013

3. Boeynaems S, Bogaert E, Michiels E, Gijselinck I, Sieben A, Jovicic A et al (2016) Drosophila screen connects nuclear transport genes to DPR pathology in c9ALS/FTD. Sci Rep 6:20877. https://doi.org/10.1038/srep20877

4. Brill MS, Kleele T, Ruschkies L, Wang M, Marahori NA, Reuter MS et al (2016) Branch-specific microtubule destabilization mediates axon branch loss during neuromuscular synapse elimination. Neuron 92:845-856. https://doi.org/10.1016/j.neuron.2016.09.049

5. Capasso M, Anzellotti F, Di Giacomo R, Onofrj M (2017) Epilepsy and electroencephalographic abnormalities in C9orf72 repeat expansion. Amyotroph Lateral Scler Frontotemporal Degener 18:140-141. https://doi.org/10.1080/21678 421.2016.1231825
6. Chew J, Gendron TF, Prudencio M, Sasaguri H, Zhang YJ, Castanedes-Casey M et al (2015) Neurodegeneration. C9ORF72 repeat expansions in mice cause TDP-43 pathology, neuronal loss, and behavioral deficits. Science 348:1151-1154. https://doi. org/10.1126/science.aaa9344

7. Choi SY, Lopez-Gonzalez R, Krishnan G, Phillips HL, Li AN, Seeley WW et al (2019) C9ORF72-ALS/FTD-associated poly(GR) binds Atp5a1 and compromises mitochondrial function in vivo. Nat Neurosci 22:851-862. https://doi.org/10.1038/ s41593-019-0397-0

8. Conlon EG, Lu L, Sharma A, Yamazaki T, Tang T, Shneider NA et al (2016) The C9ORF72 GGGGCC expansion forms RNA G-quadruplex inclusions and sequesters hnRNP H to disrupt splicing in ALS brains. Elife. https://doi.org/10.7554/eLife.17820

9. Cykowski MD, Dickson DW, Powell SZ, Arumanayagam AS, Rivera AL, Appel SH (2019) Dipeptide repeat (DPR) pathology in the skeletal muscle of ALS patients with C9ORF72 repeat expansion. Acta Neuropathol 138:667-670. https://doi.org/10.1007/ s00401-019-02050-8

10. Dadon-Nachum M, Melamed E, Offen D (2011) The "dyingback" phenomenon of motor neurons in ALS. J Mol Neurosci 43:470-477. https://doi.org/10.1007/s12031-010-9467-1

11. Delacour A, Nepote V, Trumpp A, Herrera PL (2004) Nestin expression in pancreatic exocrine cell lineages. Mech Dev 121:314. https://doi.org/10.1016/j.mod.2003.11.004

12. Dobin A, Davis CA, Schlesinger F, Drenkow J, Zaleski C, Jha S et al (2013) STAR: ultrafast universal RNA-seq aligner. Bioinformatics 29:15-21. https://doi.org/10.1093/bioinformatics/bts635

13. Dubois NC, Hofmann D, Kaloulis K, Bishop JM, Trumpp A (2006) Nestin-Cre transgenic mouse line Nes-Cre1 mediates highly efficient Cre/loxP mediated recombination in the nervous system, kidney, and somite-derived tissues. Genesis 44:355-360. https://doi.org/10.1002/dvg.20226

14. Edbauer D, Haass C (2016) An amyloid-like cascade hypothesis for C9orf72 ALS/FTD. Curr Opin Neurobiol 36:99-106. https:// doi.org/10.1016/j.conb.2015.10.009

15. Finch NA, Wang X, Baker MC, Heckman MG, Gendron TF, Bieniek KF et al (2017) Abnormal expression of homeobox genes and transthyretin in C9ORF72 expansion carriers. Neurol Genet 3:e161. https://doi.org/10.1212/NXG.0000000000000161

16. Frick P, Sellier C, Mackenzie IRA, Cheng CY, Tahraoui-Bories J, Martinat C et al (2018) Novel antibodies reveal presynaptic localization of $\mathrm{C} 9$ orf72 protein and reduced protein levels in C9orf72 mutation carriers. Acta Neuropathol Commun 6:72. https://doi. org/10.1186/s40478-018-0579-0

17. Goutieres F, Aicardi J, Barth PG, Lebon P (1998) Aicardi-Goutieres syndrome: an update and results of interferon-alpha studies. Ann Neurol 44:900-907. https://doi.org/10.1002/ana.410440608

18. Guo Q, Lehmer C, Martinez-Sanchez A, Rudack T, Beck F, Hartmann H et al (2018) In situ structure of neuronal C9orf72 PolyGA aggregates reveals proteasome recruitment. Cell 172(696705):e612. https://doi.org/10.1016/j.cell.2017.12.030

19. Hammond TR, Dufort C, Dissing-Olesen L, Giera S, Young A, Wysoker A et al (2019) Single-Cell RNA sequencing of microglia throughout the mouse lifespan and in the injured brain reveals complex cell-state changes. Immunity 50(253-271):e256. https ://doi.org/10.1016/j.immuni.2018.11.004

20. Hao Z, Liu L, Tao Z, Wang R, Ren H, Sun H et al (2019) Motor dysfunction and neurodegeneration in a C9orf72 mouse line expressing poly-PR. Nat Commun 10:2906. https://doi. org/10.1038/s41467-019-10956-w

21. Hartmann H, Hornburg D, Czuppa M, Bader J, Michaelsen M, Farny D et al (2018) Proteomics and C9orf72 neuropathology identify ribosomes as poly-GR/PR interactors driving toxicity. Life Sci Alliance 1:e201800070. https://doi.org/10.26508/ lsa.201800070 
22. Hitz C, Wurst W, Kuhn R (2007) Conditional brain-specific knockdown of MAPK using Cre/loxP regulated RNA interference. Nucleic Acids Res 35:e90. https://doi.org/10.1093/nar/gkm475

23. Jiang J, Zhu Q, Gendron TF, Saberi S, McAlonis-Downes M, Seelman A et al (2016) Gain of toxicity from ALS/FTD-linked repeat expansions in C9ORF72 is alleviated by antisense oligonucleotides targeting GGGGCC-containing RNAs. Neuron 90:535550. https://doi.org/10.1016/j.neuron.2016.04.006

24. Jovicic A, Mertens J, Boeynaems S, Bogaert E, Chai N, Yamada SB et al (2015) Modifiers of C9orf72 dipeptide repeat toxicity connect nucleocytoplasmic transport defects to FTD/ALS. Nat Neurosci 18:1226-1229. https://doi.org/10.1038/nn.4085

25. Kanekura K, Yagi T, Cammack AJ, Mahadevan J, Kuroda M, Harms MB et al (2016) Poly-dipeptides encoded by the C9ORF72 repeats block global protein translation. Hum Mol Genet 25:18031813. https://doi.org/10.1093/hmg/ddw052

26. Keren-Shaul H, Spinrad A, Weiner A, Matcovitch-Natan O, Dvir-Szternfeld R, Ulland TK et al (2017) A unique microglia type associated with restricting development of Alzheimer's disease. Cell 169(1276-1290):e1217. https://doi.org/10.1016/j. cell.2017.05.018

27. Kerschensteiner M, Reuter MS, Lichtman JW, Misgeld T (2008) Ex vivo imaging of motor axon dynamics in murine triangularis sterni explants. Nat Protoc 3:1645-1653. https:// doi.org/10.1038/nprot.2008.160

28. Khosravi B, Hartmann H, May S, Mohl C, Ederle H, Michaelsen $\mathrm{M}$ et al (2017) Cytoplasmic poly-GA aggregates impair nuclear import of TDP-43 in C9orf72 ALS/FTLD. Hum Mol Genet 26:790-800. https://doi.org/10.1093/hmg/ddw432

29. Khosravi B, LaClair KD, Riemenschneider H, Zhou Q, Frottin $\mathrm{F}$, Mareljic N et al (2020) Cell-to-cell transmission of C9orf72 poly-(Gly-Ala) triggers key features of ALS/FTD. EMBO J 39:e102811. https://doi.org/10.15252/embj.2019102811

30. Kim HJ, Kim NC, Wang YD, Scarborough EA, Moore J, Diaz $\mathrm{Z}$ et al (2013) Mutations in prion-like domains in hnRNPA2B1 and hnRNPA1 cause multisystem proteinopathy and ALS. Nature 495:467-473. https://doi.org/10.1038/nature11922

31. Kwon I, Xiang S, Kato M, Wu L, Theodoropoulos P, Wang T et al (2014) Poly-dipeptides encoded by the C9orf72 repeats bind nucleoli, impede RNA biogenesis, and kill cells. Science 345:1139-1145. https://doi.org/10.1126/science.1254917

32. Lee KH, Zhang P, Kim HJ, Mitrea DM, Sarkar M, Freibaum BD et al (2016) C9orf72 dipeptide repeats impair the assembly, dynamics, and function of membrane-less organelles. Cell 167(774-788):e717. https://doi.org/10.1016/j.cell.2016.10.002

33. Lee YB, Chen HJ, Peres JN, Gomez-Deza J, Attig J, Stalekar M et al (2013) Hexanucleotide repeats in ALS/FTD form lengthdependent RNA foci, sequester RNA binding proteins, and are neurotoxic. Cell Rep 5:1178-1186. https://doi.org/10.1016/j. celrep.2013.10.049

34. Liu Y, Pattamatta A, Zu T, Reid T, Bardhi O, Borchelt DR et al (2016) C9orf72 BAC mouse model with motor deficits and neurodegenerative features of ALS/FTD. Neuron 90:521-534. https://doi.org/10.1016/j.neuron.2016.04.005

35. Lopez-Gonzalez R, Lu Y, Gendron TF, Karydas A, Tran H, Yang D et al (2016) Poly(GR) in C9ORF72-related ALS/FTD compromises mitochondrial function and increases oxidative stress and DNA damage in iPSC-derived motor neurons. Neuron 92:383-391. https://doi.org/10.1016/j.neuron.2016.09.015

36. Love MI, Huber W, Anders S (2014) Moderated estimation of fold change and dispersion for RNA-seq data with DESeq2. Genome Biol 15:550. https://doi.org/10.1186/s1305 9-014-0550-8

37. Mackenzie IR, Arzberger T, Kremmer E, Troost D, Lorenzl $\mathrm{S}$, Mori K et al (2013) Dipeptide repeat protein pathology in C9ORF72 mutation cases: clinico-pathological correlations.
Acta Neuropathol 126:859-879. https://doi.org/10.1007/s0040 1-013-1181-y

38. Mackenzie IR, Frick P, Grasser FA, Gendron TF, Petrucelli L, Cashman NR et al (2015) Quantitative analysis and clinicopathological correlations of different dipeptide repeat protein pathologies in C9ORF72 mutation carriers. Acta Neuropathol 130:845-861. https://doi.org/10.1007/s00401-015-1476-2

39. Malaguarnera L, Musumeci M, Di Rosa M, Scuto A, Musumeci $S$ (2005) Interferon-gamma, tumor necrosis factor-alpha, and lipopolysaccharide promote chitotriosidase gene expression in human macrophages. J Clin Lab Anal 19:128-132. https://doi. org/10.1002/jcla.20063

40. Mathys H, Adaikkan C, Gao F, Young JZ, Manet E, Hemberg M et al (2017) Temporal tracking of microglia activation in neurodegeneration at single-cell resolution. Cell Rep 21:366-380. https ://doi.org/10.1016/j.celrep.2017.09.039

41. May S, Hornburg D, Schludi MH, Arzberger T, Rentzsch K, Schwenk BM et al (2014) C9orf72 FTLD/ALS-associated Gly-Ala dipeptide repeat proteins cause neuronal toxicity and Unc119 sequestration. Acta Neuropathol 128:485-503. https:// doi.org/10.1007/s00401-014-1329-4

42. Minatohara K, Akiyoshi M, Okuno H (2015) Role of immediateearly genes in synaptic plasticity and neuronal ensembles underlying the memory trace. Front Mol Neurosci 8:78. https://doi. org/10.3389/fnmol.2015.00078

43. Mizielinska S, Gronke S, Niccoli T, Ridler CE, Clayton EL, Devoy A et al (2014) C9orf72 repeat expansions cause neurodegeneration in Drosophila through arginine-rich proteins. Science 345:1192-1194. https://doi.org/10.1126/science.1256800

44. Moens TG, Niccoli T, Wilson KM, Atilano ML, Birsa N, Gittings LM et al (2019) C9orf72 arginine-rich dipeptide proteins interact with ribosomal proteins in vivo to induce a toxic translational arrest that is rescued by eIF1A. Acta Neuropathol 137:487-500. https://doi.org/10.1007/s00401-018-1946-4

45. Mori K, Arzberger T, Grasser FA, Gijselinck I, May S, Rentzsch $\mathrm{K}$ et al (2013) Bidirectional transcripts of the expanded C9orf72 hexanucleotide repeat are translated into aggregating dipeptide repeat proteins. Acta Neuropathol 126:881-893. https://doi. org/10.1007/s00401-013-1189-3

46. Mori K, Weng SM, Arzberger T, May S, Rentzsch K, Kremmer E et al (2013) The C9orf72 GGGGCC repeat is translated into aggregating dipeptide-repeat proteins in FTLD/ALS. Science 339:1335-1338. https://doi.org/10.1126/science.1232927

47. Neumann M, Sampathu DM, Kwong LK, Truax AC, Micsenyi MC, Chou TT et al (2006) Ubiquitinated TDP-43 in frontotemporal lobar degeneration and amyotrophic lateral sclerosis. Science 314:130-133. https://doi.org/10.1126/science.1134108

48. Nonaka T, Masuda-Suzukake M, Hosokawa M, Shimozawa A, Hirai S, Okado H et al (2018) C9ORF72 dipeptide repeat poly-GA inclusions promote intracellular aggregation of phosphorylated TDP-43. Hum Mol Genet 27:2658-2670. https://doi.org/10.1093/ hmg/ddy 174

49. O'Rourke JG, Bogdanik L, Muhammad AK, Gendron TF, Kim $\mathrm{KJ}$, Austin A et al (2015) C9orf72 BAC transgenic mice display typical pathologic features of ALS/FTD. Neuron 88:892-901. https://doi.org/10.1016/j.neuron.2015.10.027

50. Oeckl P, Weydt P, Steinacker P, Anderl-Straub S, Nordin F, Volk AE et al (2019) Different neuroinflammatory profile in amyotrophic lateral sclerosis and frontotemporal dementia is linked to the clinical phase. J Neurol Neurosurg Psychiatry 90:4-10. https ://doi.org/10.1136/jnnp-2018-318868

51. Olesen MN, Wuolikainen A, Nilsson AC, Wirenfeldt M, Forsberg $\mathrm{K}$, Madsen JS et al (2020) Inflammatory profiles relate to survival in subtypes of amyotrophic lateral sclerosis. Neurol Neuroimmunol Neuroinflamm. https://doi.org/10.1212/NXI.0000000000 000697 
52. Peter RS, Rosenbohm A, Dupuis L, Brehme T, Kassubek J, Rothenbacher D et al (2017) Life course body mass index and risk and prognosis of amyotrophic lateral sclerosis: results from the ALS registry Swabia. Eur J Epidemiol 32:901-908. https://doi. org/10.1007/s10654-017-0318-z

53. Peters OM, Cabrera GT, Tran H, Gendron TF, McKeon JE, Metterville J et al (2015) Human C9ORF72 hexanucleotide expansion reproduces RNA foci and dipeptide repeat proteins but not neurodegeneration in BAC transgenic mice. Neuron 88:902-909. https://doi.org/10.1016/j.neuron.2015.11.018

54. Rohrer JD, Nicholas JM, Cash DM, van Swieten J, Dopper E, Jiskoot L et al (2015) Presymptomatic cognitive and neuroanatomical changes in genetic frontotemporal dementia in the Genetic Frontotemporal dementia Initiative (GENFI) study: a cross-sectional analysis. Lancet Neurol 14:253-262. https://doi. org/10.1016/S1474-4422(14)70324-2

55. Saberi S, Stauffer JE, Jiang J, Garcia SD, Taylor AE, Schulte D et al (2018) Sense-encoded poly-GR dipeptide repeat proteins correlate to neurodegeneration and uniquely co-localize with TDP43 in dendrites of repeat-expanded C9orf72 amyotrophic lateral sclerosis. Acta Neuropathol 135:459-474. https://doi.org/10.1007/ s00401-017-1793-8

56. Sala Frigerio C, Wolfs L, Fattorelli N, Thrupp N, Voytyuk I, Schmidt I et al (2019) The major risk factors for Alzheimer's disease: age, sex, and genes modulate the microglia response to abeta plaques. Cell Rep 27(1293-1306):e1296. https://doi. org/10.1016/j.celrep.2019.03.099

57. Schludi MH, Becker L, Garrett L, Gendron TF, Zhou Q, Schreiber $F$ et al (2017) Spinal poly-GA inclusions in a C9orf72 mouse model trigger motor deficits and inflammation without neuron loss. Acta Neuropathol 134:241-254. https://doi.org/10.1007/ s00401-017-1711-0

58. Schludi MH, May S, Grasser FA, Rentzsch K, Kremmer E, Kupper $C$ et al (2015) Distribution of dipeptide repeat proteins in cellular models and C9orf72 mutation cases suggests link to transcriptional silencing. Acta Neuropathol 130:537-555. https://doi. org/10.1007/s00401-015-1450-Z

59. Steinacker P, Verde F, Fang L, Feneberg E, Oeckl P, Roeber S et al (2018) Chitotriosidase (CHIT1) is increased in microglia and macrophages in spinal cord of amyotrophic lateral sclerosis and cerebrospinal fluid levels correlate with disease severity and progression. J Neurol Neurosurg Psychiatry 89:239-247. https:// doi.org/10.1136/jnnp-2017-317138

60. Tam OH, Rozhkov NV, Shaw R, Kim D, Hubbard I, Fennessey $\mathrm{S}$ et al (2019) Postmortem cortex samples identify distinct molecular subtypes of ALS: retrotransposon activation, oxidative stress, and activated glia. Cell Rep 29:1164-1177. https://doi. org/10.1016/j.celrep.2019.09.066
61. Tronche F, Kellendonk C, Kretz O, Gass P, Anlag K, Orban PC et al (1999) Disruption of the glucocorticoid receptor gene in the nervous system results in reduced anxiety. Nat Genet 23:99-103. https://doi.org/10.1038/12703

62. Wen X, Tan W, Westergard T, Krishnamurthy K, Markandaiah SS, Shi Y et al (2014) Antisense proline-arginine RAN dipeptides linked to C9ORF72-ALS/FTD form toxic nuclear aggregates that initiate in vitro and in vivo neuronal death. Neuron 84:1213-1225. https://doi.org/10.1016/j.neuron.2014.12.010

63. White MR, Mitrea DM, Zhang P, Stanley CB, Cassidy DE, Nourse A et al (2019) C9orf72 Poly(PR) dipeptide repeats disturb biomolecular phase separation and disrupt nucleolar function. Mol Cell 74(713-728):e716. https://doi.org/10.1016/j.molcel.2019.03.019

64. Yin P, Guo X, Yang W, Yan S, Yang S, Zhao T et al (2019) Caspase-4 mediates cytoplasmic accumulation of TDP-43 in the primate brains. Acta Neuropathol 137:919-937. https://doi. org/10.1007/s00401-019-01979-0

65. Yousef A, Robinson JL, Irwin DJ, Byrne MD, Kwong LK, Lee EB et al (2017) Neuron loss and degeneration in the progression of TDP-43 in frontotemporal lobar degeneration. Acta Neuropathol Commun 5:68. https://doi.org/10.1186/s40478-017-0471-3

66. Zhang YJ, Gendron TF, Ebbert MTW, O'Raw AD, Yue M, JansenWest K et al (2018) Poly(GR) impairs protein translation and stress granule dynamics in C9orf72-associated frontotemporal dementia and amyotrophic lateral sclerosis. Nat Med 24:11361142. https://doi.org/10.1038/s41591-018-0071-1

67. Zhang YJ, Gendron TF, Grima JC, Sasaguri H, Jansen-West K, $\mathrm{Xu}$ YF et al (2016) C9ORF72 poly(GA) aggregates sequester and impair HR23 and nucleocytoplasmic transport proteins. Nat Neurosci 19:668-677. https://doi.org/10.1038/nn.4272

68. Zhang YJ, Guo L, Gonzales PK, Gendron TF, Wu Y, Jansen-West $\mathrm{K}$ et al (2019) Heterochromatin anomalies and double-stranded RNA accumulation underlie C9orf72 poly(PR) toxicity. Science. https://doi.org/10.1126/science.aav2606

69. Zhou Q, Lehmer C, Michaelsen M, Mori K, Alterauge D, Baumjohann D et al (2017) Antibodies inhibit transmission and aggregation of C9orf72 poly-GA dipeptide repeat proteins. EMBO Mol Med. https://doi.org/10.15252/emmm.201607054

70. Zu T, Liu Y, Banez-Coronel M, Reid T, Pletnikova O, Lewis J et al (2013) RAN proteins and RNA foci from antisense transcripts in C9ORF72 ALS and frontotemporal dementia. Proc Natl Acad Sci USA 110:E4968-4977. https://doi.org/10.1073/pnas.1315438110

Publisher's Note Springer Nature remains neutral with regard to jurisdictional claims in published maps and institutional affiliations.

\section{Affiliations}

\section{Katherine D. LaClair ${ }^{1} \cdot$ Qihui Zhou $^{1,2} \cdot$ Meike Michaelsen $^{1} \cdot$ Benedikt Wefers $^{1} \cdot$ Monika S. Brill $^{2,3} \cdot$ Aleksandar Janjic $^{4}$. Birgit Rathkolb ${ }^{5,6,7}$. Daniel Farny ${ }^{1} \cdot$ Mikolaj Cygan ${ }^{1}$ - Martin Hrabe de Angelis ${ }^{5,7,8}$. Wolfgang Wurst ${ }^{1,2}$. Manuela Neumann ${ }^{9,10} \cdot$ Wolfgang Enard ${ }^{4} \cdot$ Thomas Misgeld $^{1,2,3} \cdot$ Thomas Arzberger $^{1,2,11,12} \cdot$ Dieter Edbauer $^{1,2,13 \text { (D) }}$}

1 German Center for Neurodegenerative Diseases (DZNE), Munich, 81377 Munich, Germany

2 Munich Cluster for Systems Neurology (SyNergy), 80336 Munich, Germany

3 Institute of Neuronal Cell Biology, Technische Universität München, 80802 Munich, Germany
4 Department Biology II, Anthropology and Human Genomics, Ludwig-Maximilians-University Munich, 82152 Martinsried, Germany

5 German Mouse Clinic, Institute for Experimental Genetics, German Research Center for Environmental Health, Helmholtz Zentrum München, Neuherberg, Germany 
6 Institute of Molecular Animal Breeding and Biotechnology, Gene Center, Ludwig-Maximilians-University Munich, 81377 Munich, Germany

7 German Center for Diabetes Research (DZD), Ingolstädter Landstr. 1, 85764 Neuherberg, Germany

8 Chair of Experimental Genetics, School of Life Science Weihenstephan, Technische Universität München, Alte Akademie 8, 85354 Freising, Germany

9 German Center for Neurodegenerative Diseases (DZNE), Tübingen, Germany

10 Department of Neuropathology, University of Tübingen, Tübingen, Germany
11 Center for Neuropathology and Prion Research, Ludwig-Maximilians-University Munich, 81377 Munich, Germany

12 Department of Psychiatry and Psychotherapy, University Hospital, Ludwig-Maximilians-University Munich, 80336 Munich, Germany

13 Graduate School of Systemic Neurosciences (GSN), Ludwig-Maximilians-University Munich, 81377 Munich, Germany 